\title{
Relaxation dynamics through a conical intersection: Quantum and quantum-classical studies
}

Carlotta Pieroni, ${ }^{1,2}$ Emanuele Marsili, ${ }^{3}$ David Lauvergnat, ${ }^{1}$ and Federica

Agostini ${ }^{\dagger 1}$

1) Université Paris-Saclay, CNRS, Institut de Chimie Physique UMR8000, 91405, Orsay, France

2) Università di Pisa, Dipartimento di Chimica e Chimica Industriale, via G. Moruzzi 13, 56124 Pisa, Italy

3)Department of Chemistry, Durham University, South Road, Durham DH1 3LE, United Kingdom

We study the relaxation process through a conical intersection of a photo-excited retinal chromophore model. The analysis is based on a two-electronic-state twodimensional Hamiltonian developed by Hahn and Stock [J. Phys. Chem. B 104 1146 (2000)] to reproduce, with a minimal model, the main features of the 11-cis to all-trans isomerization of the retinal of rhodopsin. In particular, we focus on the performance of various trajectory-based schemes to nonadiabatic dynamics, and we compare quantum-classical results to numerically-exact quantum vibronic wavepacket dynamics. The purpose of the work is to investigate, by analyzing electronic and nuclear observables, how the sampling of initial conditions for the trajectories affects the subsequent dynamics. 
$\dagger$ federica.agostini@universite-paris-saclay.fr

\section{INTRODUCTION}

Trajectory-based approaches to nonadiabatic dynamics are powerful tools for predicting the fate of a molecule after photo-excitation ${ }^{1-13}$ or the products of a collision reaction ${ }^{14-17}$. The underlying approximations, in the treatment of nuclear dynamics $3,12,18-20$ and in the description of electron-nuclear coupling ${ }^{12,21-25}$, make them computationally efficient, at least compared to a numerically-exact solution of the time-dependent Schrödinger equation ${ }^{19}$. Clearly, the computational efficiency comes at the price of losing accuracy or missing critical features, such as tunnelling and zero-point energy ${ }^{26-30}$, interferences ${ }^{31,32}$, quantum decoherence ${ }^{33-36}$, to name some of the usual suspects. Related to this point, we recently reported a combined quantum and quantum-classical, i.e., trajectory-based, study of the photo-isomerization process in a retinal chromophore model $^{37}$. There, we identified some issues of various quantum-classical methods in correctly predicting the energy redistribution from electrons to nuclear vibrations during the ultrafast relaxation dynamics through conical intersections, after energy is pumped into the system via UV photoexcitation. The purpose of the present work is to investigate further this problem and, possibly, to shed some light on its origin.

We focus our analysis on a two-electronic-state two-dimensional model for the photo-induced 11-cis to all-trans isomerization of retinal in rhodopsin ${ }^{38}$ (a simplified, and well-established, precursor of the model $^{39}$ studied in Ref. ${ }^{37}$ ). The effective nuclear modes defining the model are an angular reaction coordinate and a collective vibration characterizing "a delocalized stretching motion of the polyene chain, whereby single and double bonds interchange ${ }^{\prime \prime 38}$. Electronic ground $\left(S_{0}\right)$ and first-excited $\left(S_{1}\right)$ singlet states present a conical intersection at some geometry, as will be shown in the following. The reduced dimensionality of the model, and the fact that it is parametrized to reproduce features observed in Raman and time-resolved experiments ${ }^{38}$, made it for long time the preferred choice to study the ultrafast relaxation process of the photo-excited retinal based on quantum-dynamical simulations ${ }^{11,40-51}$. To our knowledge, however, trajectory-based methods have not yet been employed, systematically, in combination with this model ${ }^{44,49,52}$. The interest in carrying out such a study clearly lies in the possibility of benchmarking quantum-classical results against vibronic wavepacket dynamics with the same "force field", allowing to identify the potential issues of the approximated approaches (as shown in Ref. ${ }^{37}$ ).

The trajectory-based methods under study in this work are trajectory surface hopping $(\mathrm{TSH})^{53}$, tra- 
jectory surface hopping including energy decoherence corrections (TSH-EDC) ${ }^{54}$, Ehrenfest dynamics $(\mathrm{EH})^{33,55}$, and the coupled-trajectory mixed quantum-classical (CT-MQC) ${ }^{56-59}$ scheme derived from the exact factorization of the time-dependent electron-nuclear wavefunction ${ }^{60-62}$. Each of the methods mentioned here have its strengths and drawbacks: TSH suffers from overcoherence, but it is very intuitive and easy to implement ${ }^{4}$; TSH-EDC improves on TSH including decoherence effects, but the correction might be insufficient for some systems ${ }^{37}$; the mean-field nature of $\mathrm{EH}$ dynamics lacks the capability of reproducing diverging reactions paths in nuclear space, but it is computationally very efficient ${ }^{5,63}$; CT-MQC accounts for decoherence effects related to the branching of paths in nuclear space, but it is numerically costly due to the coupling of trajectories ${ }^{64}$. While these methods suggest well-defined procedures to propagate classical-like trajectories coupled to electronic nonadiabatic dynamics, the choice of initial conditions is not specified. Often, initial positions and momenta are Wigner-sampled ${ }^{65-69}$, in order to account for quantum effects, such as zero-point energy, via a quantum-like selection of initial conditions, but usually this is done in the harmonic approximation. Moreover, due to the fact that trajectories are then evolved in a classical-like way, the question might arise as to whether the inconsistency between preparation of "quantum" initial conditions and evolution of "classical" trajectories is responsible for inaccuracies that can be circumvented with a more appropriate choice of initial sampling.

The present study on initial conditions sampling schemes places itself in the context of greater efforts of different communities that focus on the topic of quantum molecular dynamics simulations. Depending on the fundamental theory which the trajectory-based schemes are based on, various formulations of the problem, as well as solutions, have been proposed over the years. Real-time and imaginary-time propagators, for example, lend themselves to a representation in terms of path integrals, that can be approximated semiclassically ${ }^{70}$, linearized ${ }^{71,72}$, or expressed with mapping variables ${ }^{73,74}$. There, the problems of sampling initial conditions, Wigner distribution, and mapping variables have been discussed. Recently, the sampling of initial conditions has been discussed in the context of excited-state dynamics, and in connection with the "setup" of the photo-excitation ${ }^{75}$, thus explicitly accounting for the effect of the external time-dependent field that initiates the dynamics. Moreover, it is worth mentioning that, in the context of condensed-phase simulations, great effort has been, and currently is, devoted to address some related issues. Examples are the sampling of the thermal Wigner function at equilibrium for the calculation of time correlation functions $^{76-79}$, the preservation of the probability distribution along classical dynamics ${ }^{80}$, or zero-point energy leakage ${ }^{81}$.

The examples just presented clearly show that the literature on the topic of sampling positions and mo- 
menta for trajectory-based schemes is vast, and our work (simply) aims at studying this topic in relation with trajectory surface hopping, Ehrenfest dynamics, and the exact factorization. The common feature of the selected method is that, they are all designed to propagate (with trajectories) nuclear wavefunctions, and not, for instance, the density matrix, in the presence of electronic nonadiabatic effects. Therefore, the purpose of this work is to investigate the relaxation process through a conical intersection of the photoexcited (model of) retinal via quantum and quantum-classical dynamics, to test different schemes for sampling initial positions and momenta for the trajectories, and to analyze the effect of the sampling on the subsequent electronic and nuclear dynamics. The model and the techniques employed in this work are described in Section II. Section III presents the results by analyzing the dynamics and the effect of different ways of sampling the initial conditions. Our conclusions are reported in Section IV.

\section{DESCRIPTION OF THE MODEL AND OF THE DYNAMICS TECHNIQUES}

The photo-induced dynamics and cis-trans isomerization process of the retinal in rhodospin is described by the $2 \times 2$ Hamiltonian given in the diabatic basis as ${ }^{38}$

$$
\hat{H}=\frac{\hat{P}_{\varphi}^{2}}{2 m}+\frac{\hat{P}_{q}^{2}}{2 \omega^{-1}}+\left(\begin{array}{cc}
V_{00}(\varphi, q) & V_{01}(q) \\
V_{10}(q) & V_{11}(\varphi, q)
\end{array}\right) .
$$

Here, nuclear - effective - modes are the (dimensionless) reactive angular coordinate $\varphi$ and the collective vibration $q$. Two electronic states are considered, thus the "electronic" Hamiltonian - later on indicated as $\hat{H}_{e l}(\varphi, q)-$ is given as a $2 \times 2$ matrix whose elements are

$$
\begin{aligned}
& V_{00}(\varphi, q)=\frac{1}{2} W_{0}(1-\cos \varphi)+\frac{1}{2} \omega q^{2} \\
& V_{11}(\varphi, q)=E_{1}-\frac{1}{2} W_{1}(1-\cos \varphi)+\frac{1}{2} \omega q^{2}+\kappa q \\
& V_{01}(q)=V_{10}(q)=\lambda q
\end{aligned}
$$

and depend on nuclear coordinates. The stretching mode is also called coupling mode, as the potential coupling $V_{10}$ only depends on $q$. Nuclear kinetic energy is expressed via the momentum operators along $\varphi$ and $q$, where $\hat{P}_{\varphi}^{2}$ and $\hat{P}_{q}^{2}$ are simply second-order derivatives with respect to $\varphi$ and $q$, respectively. The model is parametrized as follows: the effective mass of the reaction coordinate $m^{-1}=4.84 \cdot 10^{-4} \mathrm{eV}$ is chosen so as to achieve isomerization within $200 \mathrm{fs}$; the frequency of the coupling mode is $\omega=0.19 \mathrm{eV}$ and the interstate coupling is $\lambda=0.19 \mathrm{eV}$, with $\kappa=0.1 \mathrm{eV}$ indicating a gradient in the excited state; the parameters $W_{0}=3.6 \mathrm{eV}, W_{1}=1.09 \mathrm{eV}$ and $E_{1}=2.48 \mathrm{eV}$ are chosen in order to match the $S_{0} / S_{1}$ 
gap to the center frequency of the absorption bands of the cis and trans isomers. Diagonalization of the electronic Hamiltonian yields the adiabatic potential energy surfaces (PESs) $S_{0}$ and $S_{1}$, presenting a conical intersection at $\varphi_{C I} \simeq \pm \frac{\pi}{2}, q_{C I}=0$. At the cis geometry $\left(\varphi_{\text {cis }}=0\right)$, the excitation energy $\Delta E_{e x}$ to induce a $S_{0}$-to- $S_{1}$ transition at $q=0$ lies in the visible domain, being $\Delta E_{e x}=2.48 \mathrm{eV}\left(\lambda_{e x}=500 \mathrm{~nm}\right)$.

Photo-dynamics is initiated by an instantaneous $S_{0}$-to- $S_{1}$ transition that promotes the ground-state wavepacket to the excited state without structural rearrangements. The ground-state wavepacket is a twodimensional real Gaussian of the form

$$
\chi_{S_{0}}(\varphi, q, t=0)=\sqrt[4]{\frac{1}{\pi \sigma_{\varphi}^{2}}} \exp \left[-\frac{\varphi^{2}}{2 \sigma_{\varphi}^{2}}\right] \sqrt[4]{\frac{1}{\pi \sigma_{q}^{2}}} \exp \left[-\frac{q^{2}}{2 \sigma_{q}^{2}}\right]
$$

with $\sigma_{\varphi}=0.128$ and $\sigma_{q}=1.0$. The nuclear (probability) density at all times is given as the sum of the $S_{0}$ and $S_{1}$ (probability) densities

$$
|\chi(\varphi, q, t)|^{2}=\left|\chi_{S_{0}}(\varphi, q, t)\right|^{2}+\left|\chi_{S_{1}}(\varphi, q, t)\right|^{2}
$$

The probability for the system to be in the electronic ground $\left(S_{0}\right)$ or excited $\left(S_{1}\right)$ state is determined as the integral of the corresponding nuclear density,

$$
\rho_{S_{k}}(t)=\iint\left|\chi_{S_{k}}(\varphi, q, t)\right|^{2} d \varphi d q \quad \text { with } k=0,1
$$

which we will also refer to as "electronic population". This electronic population can be decomposed into two contributions as $\rho_{S_{k}}(t)=P_{S_{k}}^{\text {cis }}(t)+P_{S_{k}}^{\text {trans }}(t)$, from the cis and trans conformers,

$$
\begin{aligned}
P_{S_{k}}^{\text {cis }}(t) & =\int_{|\varphi|<\frac{\pi}{2}} \int\left|\chi_{S_{k}}(\varphi, q, t)\right|^{2} d \varphi d q \\
P_{S_{k}}^{\text {trans }}(t) & =\int_{|\varphi|>\frac{\pi}{2}} \int\left|\chi_{S_{k}}(\varphi, q, t)\right|^{2} d \varphi d q,
\end{aligned}
$$

yielding the probability of each conformer in each state. Average nuclear kinetic energy is determined as the expectation value of the operators $\hat{P}_{\varphi}^{2} /(2 m)$ and $\hat{P}_{q}^{2} /\left(2 \omega^{-1}\right)$ in Eq. (1) over the vibronic wavefunction.

Vibronic wavepacket dynamics after photo-excitation is simulated in the diabatic basis using ElVi$\mathrm{bRot}^{82}$, whose results are used as benchmark for the trajectory-based methods. The diabatic densities are transformed to the adiabatic representation for the comparison with the trajectory-based approaches. The nuclear wavepacket has been expanded in a two-dimensional basis set formed by the direct product of two uni-dimensional basis sets for each diabatic state. The harmonic-oscillator basis set has been used for $q$ (20 basis functions), and a Fourier-series, periodic basis set has been used for $\varphi$ ( 256 basis functions); the number of grid points along each coordinate is 40 and 512, respectively for $q$ and $\varphi$. The propagation 
was performed with the Chebychev scheme for which the evolution operator is expanded on Chebychev polynomials ${ }^{83}$ with time step $1 \mathrm{fs}$. The Hamiltonian needs to be renormalized so that its spectral range lies in the interval $[-1,1]$.

For the sake of completeness, we briefly recall the fundamental aspects of TSH, TSH-EDC, EH, and CT-MQC. In all approaches, an ensemble of $N_{t r}$ trajectories needs to be propagated in order to mimic the behavior of a quantum wavepacket that potentially delocalizes and splits in configuration space. Henceforth, the symbol $\mathbf{R}^{\alpha}(t)$ is used to label the trajectory $\alpha$, and indicates the values of the reaction coordinate $\varphi^{\alpha}(t)$ and of the coupling mode $q^{\alpha}(t)$ along the trajectory $\alpha$. TSH and TSH-EDC trajectories evolve according to adiabatic forces, i.e., forces that are computed from the gradients of the adiabatic PESs, namely

$$
\mathbf{F}_{\alpha}^{\mathrm{TSH}}(t)=\mathbf{F}_{\alpha}^{\mathrm{TSH}-\mathrm{EDC}}(t)=-\nabla E_{S_{*}}^{\alpha}
$$

with $S_{*}=S_{0}$ or $S_{1}$, and the symbol $E_{S_{k}}^{\alpha}(k=0,1)$ indicating the adiabatic energy at the position of the trajectory $\alpha$. At each time, the PES for the force calculation and, thus, the "force state", is selected stochastically; we employ the fewest-switches scheme for the selection and, thus, to determine the hopping probability, with velocity adjustment along the nonadiabatic coupling vectors after the hops ${ }^{53}$. EH trajectories are propagated following a "mean force", which is determined at each time from the expectation value of the electronic Hamiltonian over the instantaneous electronic state $\left|\Phi_{\mathbf{R}^{\alpha}(t)}(t)\right\rangle^{63}$,

$$
\mathbf{F}_{\alpha}^{\mathrm{EH}}(t)=\left\langle\Phi_{\mathbf{R}^{\alpha}(t)}(t)\left|\left[-\nabla \hat{H}_{e l}\left(\mathbf{R}^{\alpha}(t)\right)\right]\right| \Phi_{\mathbf{R}^{\alpha}(t)}(t)\right\rangle .
$$

In CT-MQC original algorithm ${ }^{56,57,59}$, an additional force term, if compared to the EH approach, is included as effect of the, so-called, quantum momentum. The quantum momentum keeps track of the delocalization of trajectories in configuration space by reconstructing the nuclear density as a sum of Gaussians centered at the positions of the trajectories; reconstructing the density requires that trajectories are propagated simultaneously. Each Gaussian has a fixed width all along the simulation, set to the values $\sigma_{\varphi}$ and $\sigma_{q}$ of Eq. (5). The CT-MQC force is

$$
\mathbf{F}_{\alpha}^{\mathrm{CT}-\mathrm{MQC}}(t)=\mathbf{F}_{\alpha}^{\mathrm{EH}}(t)+\mathcal{F}_{\alpha}[\underline{\mathbf{R}}(t)](t)
$$

with the additional term $\mathcal{F}_{\alpha}[\underline{\mathbf{R}}(t)](t)$ depending on the positions of all trajectories, indicated with the underlined symbol $\underline{\mathbf{R}}(t)$ and on the adiabatic forces accumulated over time along the trajectories (see Section II A for a detailed discussion).

The electronic time-dependent state of the system $\left|\Phi_{\mathbf{R}^{\alpha}(t)}(t)\right\rangle$ along a trajectory $\mathbf{R}^{\alpha}(t)$ is expressed as a 
linear combination of adiabatic states $\left\{\left|\phi_{\mathbf{R}^{\alpha}(t)}^{S_{k}}\right\rangle\right\}_{k=0,1}$

$$
\left|\Phi_{\mathbf{R}^{\alpha}(t)}(t)\right\rangle=C_{S_{0}}\left(\mathbf{R}^{\alpha}(t), t\right)\left|\phi_{\mathbf{R}^{\alpha}(t)}^{S_{0}}\right\rangle+C_{S_{1}}\left(\mathbf{R}^{\alpha}(t), t\right)\left|\phi_{\mathbf{R}^{\alpha}(t)}^{S_{1}}\right\rangle .
$$

In the more general CT-MQC approach, the electronic coefficients just introduced $C_{S_{k}}\left(\mathbf{R}^{\alpha}(t), t\right)(k=0,1)$ depend implicitly on time via their dependence on the trajectory; the other methods make the assumptions that those coefficients depend on time only explicitly, thus the appropriate notation should be $C_{S_{k}}^{\alpha}(t)$. To simplify the following equations, we will use this symbol for CT-MQC as well, although the implicit dependence on time has been considered in the derivation of the evolution equation ${ }^{56,57,59}$. Electronic coefficients in $\mathrm{TSH}(-\mathrm{EDC})$ and $\mathrm{EH}$ evolve in time, together with the trajectory $\alpha$, according to

$$
\dot{C}_{S_{k}}^{\alpha}(t)=-\frac{i}{\hbar} E_{S_{k}}^{\alpha} C_{S_{k}}^{\alpha}(t)-\dot{\mathbf{R}}^{\alpha}(t) \cdot \mathbf{d}_{S_{k} S_{l}}^{\alpha} C_{S_{l}}^{\alpha}(t)
$$

The symbol $E_{S_{k}}^{\alpha}$ stands for the value of the adiabatic energy $S_{k}$ at the position of the trajectory $\alpha, \mathbf{d}_{S_{k} S_{l}}^{\alpha}$ is the nonadiabatic coupling vector between states $S_{k}$ and $S_{l}$ at the position of the trajectory $\alpha$, the velocity of the trajectory is indicated as $\dot{\mathbf{R}}^{\alpha}(t)$. Note that in Eq. (14), the term containing the nonadiabatic coupling vector is non-zero if $k \neq l$, and in general - with more than two electronic states - a sum over $l$ appears. In TSH-EDC, the electronic coefficient that is not associated to the force state is damped exponentially over a characteristic decoherence time $\tau_{S_{k} S_{l}}^{\alpha}=\left(1+\mathcal{C} / \mathcal{K}^{\alpha}\right) /\left|E_{S_{k}}^{\alpha}-E_{S_{l}}^{\alpha}\right|$ (with $\mathcal{K}^{\alpha}$ the kinetic energy of the trajectory $\alpha$ and $\mathcal{C}=0.2721 \mathrm{eV})$. In CT-MQC electronic equation, an additional term is present if compared to Eq. (14), namely

$$
\dot{C}_{S_{k}}^{\alpha}(t)=\left(-\frac{i}{\hbar} E_{S_{k}}^{\alpha}+\mathcal{D}_{S_{k}}^{\alpha}(t)\right) C_{S_{k}}^{\alpha}(t)-\dot{\mathbf{R}}^{\alpha}(t) \cdot \mathbf{d}_{S_{k} S_{l}}^{\alpha} C_{S_{l}}^{\alpha}(t)
$$

The additional (real) term in parenthesis, $\mathcal{D}_{S_{k}}^{\alpha}(t)$, depends on the quantum momentum, on the timedependent vector potential of the theory, and on an adiabatic $\left(S_{k}\right)$ force accumulated over time along the trajectory $\alpha$. Note that, a regularization scheme was introduced in the derivation of Eq. (15) starting from the exact-factorization equations $s^{56,57,59}$, to ensure that there is no population transfer between two electronic states if the nonadiabatic coupling vectors are zero.

In TSH and in TSH-EDC, electronic population in state $S_{k}$ at a certain time $t$ is estimated as the ratio of the number of trajectories in that state at that time over the total number of trajectories $N_{t r}$; in EH and CTMQC, the same information is obtained as the average over all trajectories of $\left|C_{S_{k}}^{\alpha}(t)\right|^{2}$. The population in each state can be decomposed into cis and trans probabilities, similarly to Eqs. (8) and (9), by selecting in the trajectory count the $\alpha^{\prime}$ s that satisfy the conditions $\left|\varphi^{\alpha}(t)\right|<\frac{\pi}{2}$ (cis) or $\left|\varphi^{\alpha}(t)\right|>\frac{\pi}{2}$ (trans), since $\varphi \in[-\pi, \pi]$. Nuclear kinetic energy is estimated as the average over the trajectories of the quantities $\left(P_{\varphi}^{\alpha}(t)\right)^{2} /(2 m)$ and 
$\left(P_{q}^{\alpha}(t)\right)^{2} /\left(2 \omega^{-1}\right)$, where $P_{\varphi}^{\alpha}(t)$ and $P_{q}^{\alpha}(t)$ are the classical momenta of the trajectory $\alpha$ at time $t$ along the modes $\varphi$ and $q$, respectively.

Quantum-classical dynamics is performed in the adiabatic basis, with adiabatic PESs determined by diagonalizing the electronic Hamiltonian in Eq. (1). The nonadiabatic couplings are computed analytically with the usual Hellmann-Feynman theorem ${ }^{84}$ from the eigenvectors of the electronic Hamiltonian in Eq. (1). This procedure is implemented in the ModelLib library ${ }^{85}$. For EH and CT-MQC ${ }^{86}$, we use $N_{t r}=1000$. In TSH and TSH-EDC, in order to account for the stochastic nature of the hopping algorithm, each trajectory of the $N_{t r}$ is propagated with 10 different jump histories, for a total of 10000 trajectories. The velocityVerlet algorithm is used to integrate classical nuclear equations, and the fourth-order Runge-Kutta method is used for the electronic evolution; nuclear and electronic dynamics are integrated with at time step of $0.0024 \mathrm{fs}(0.1 \mathrm{au})$. The electronic initial condition is chosen as $C_{S_{0}}^{\alpha}(t=0)=(0.0,0.0), C_{S_{1}}^{\alpha}(t=0)=(1.0,0.0)$ $\forall \alpha$.

\section{A. Some observations on CT-MQC}

The CT-MQC algorithm has been recently derived in the context of the exact factorization ${ }^{4,35,56,57}$. Thus, we devote this section to a brief analysis in the context of previously-derived approaches, and to a discussion that highlights its strengths and limitations.

The main feature of CT-MQC is the possibility to account for decoherence effects via the quantum momentum. Interestingly, the quantum momentum has been recently "rediscovered" within the exact factorization, but it has a longer history in the context of the quantum-trajectory formalism 87,88 - together with the idea of reconstructing the nuclear density as a combination of Gaussians to compute the quantum momentum ${ }^{89}$. However, the quantum momentum in the exact factorization appears in the electronic evolution equation, and it is not related to the quantum potential of the quantum-trajectory formalism (which is actually completely neglected in the derivation of CT-MQC); it is only in the quantum-classical version of the exact-factorization equations that the quantum momentum emerges in the expression of the force. In the expression of the classical force given in Eq. (12), the additional term that depends on the quantum momentum $\mathcal{Q}_{\alpha}(t)$ is

$$
\mathcal{F}_{\alpha}[\underline{\mathbf{R}}(t)](t)=\left|C_{S_{0}}^{\alpha}(t)\right|^{2}\left|C_{S_{1}}^{\alpha}(t)\right|^{2}\left[\mathbf{f}_{\alpha ; S_{0}}(t)-\mathbf{f}_{\alpha ; S_{1}}(t)\right]\left(\frac{2 \mathcal{Q}_{\alpha}(t)}{\hbar \mathbf{M}} \cdot\left[\mathbf{f}_{\alpha ; S_{0}}(t)-\mathbf{f}_{\alpha ; S_{1}}(t)\right]\right) .
$$

This expression is given for the particular two-state model studied here; $\mathbf{f}_{\alpha ; S_{k}}(t)$ is the adiabatic force from the PES $S_{k}$ accumulated over time along the trajectory $\alpha ; \mathbf{M}$ is the diagonal mass matrix. The effect of this 
quantum-momentum force is usually localized, in time and space, since the product of the populations of the electronic states along a trajectory is often negligible. It can become important after the crossing of a nonadiabatic region (conical intersection or avoided crossing) when the populations become different from 0 and 1 . When portions of the nuclear density separate in space and coherence is lost, $\mathcal{F}_{\alpha}[\underline{\mathbf{R}}(t)](t)$ tends rapidly to zero (see previous work ${ }^{21,57,62,90}$ on the exact factorization based on exact dynamics and analytical studies). Therefore, CT-MQC equations have been derived under the assumption that decoherence effects, induced by the quantum momentum, (i) become important following the passage of the nuclear wavepacket through a region of localized nonadiabatic coupling, but (ii) rapidly disappear as consequence of the spatial splitting of the nuclear density along diverging paths. The latter is not fully consistent with the observed dynamics, in fact, as will be shown in Section III, the studied system dynamics does not yield complete decoherence, because the $S_{0}$ and $S_{1}$ wavepackets become completely delocalized in the available (two-dimensional) configuration space. In addition, nuclear dynamics for this particular model system is very quantum ${ }^{44}$, in the sense that the density delocalizes in space and develops interference patterns. Therefore, accurately reproducing the nuclear density from the knowledge of the positions of the trajectories, to compute the quantum momentum, becomes challenging, and the results are very noisy. To circumvent numerical instabilities that are mainly related to the assumptions made in the derivation of CTMQC, but not fully consistent with the simulated dynamics, the results reported in Section III have been obtained by completely neglecting $\mathcal{F}_{\alpha}[\underline{\mathbf{R}}(t)](t)$ in Eq. (12). We expect that for a more classical behavior of a quantum wavepacket and for higher dimensions, as we have previously shown ${ }^{4}$, the assumptions that CT-MQC is based on are consistent with the actual dynamics of the system. An alternative strategy would be to relax the assumptions (i) and (ii) made in the derivation of CT-MQC, and propose a slightly modified version of the algorithm. Such an alternative is, however, beyond the scope of the present study. Note that the "instabilities" just discussed are not related to the well-studied instabilities $29,91,92$ of the quantum force encountered in the context of the quantum-trajectory formalism, which have been studied as well, recently, in combination with CT-MQC and the exact factorization ${ }^{27,28}$.

Despite the fact that nuclear forces become mean-field forces, EH and CT-MQC electronic dynamics are still different, thus, the actual forces are indeed different. In fact, the quantum-momentum term in Eq. (15), namely

$$
\mathcal{D}_{S_{k}}^{\alpha}(t)=\frac{\mathcal{Q}_{\alpha}(t)}{\hbar \mathbf{M}} \cdot\left[\mathbf{f}_{\alpha, S_{k}}(t)-\mathbf{A}_{\alpha}(t)\right]
$$

is not set to zero, and decoherence effects are fully accounted for in the electronic dynamics, as we will show 
in Section III. Since the quantum momentum has to be computed for the electronic evolution equation, CTMQC trajectories remain coupled.

\section{SAMPLING OF THE INITIAL CONDITIONS}

The model and numerical approaches, i.e, exact and trajectory-based, described in Section II are employed here to test the capability of the quantum-classical methods in describing the relaxation process through a conical intersection of the photo-excited (model of) retinal. In particular, the analysis is carried out by varying the initial conditions for the trajectory schemes. The different strategies proposed here to prepare the $N_{t r}$ initial conditions are:

- $R_{q} P_{q}$ : Wigner sampling from Gaussian distributions in position space of $\varphi, q$ and momentum space of $P_{\varphi}, P_{q}$, all centered in 0 ;

- $R_{q} P_{c l}$ : sampling from Gaussian (Wigner) distributions in position space of $\varphi, q$ while trajectories are initialized with $P_{\varphi}=0, P_{q}=0$;

- $R_{c l} P_{q}$ : sampling from Gaussian (Wigner) distributions in momentum space of $P_{\varphi}, P_{q}$ while trajectories are initialized with $\varphi=0, q=0$;

- $R_{c l} P_{c l}$ : "thermal" sampling from Gaussian (Boltzmann) distributions in position space of $\varphi, q$ and momentum space of $P_{\varphi}, P_{q}$, all centered in 0 .

Wigner-sampled trajectories are more "quantum" $(q)$ as their distribution satisfies by construction the uncertainty principle (at least at the initial time), which might not be the case for the other sampling schemes, which are more "classical" $(c l)$, since positions/momenta either are set to the same value for all trajectories or are sampled from a classical (Boltzmann) distribution. In the procedure labelled $R_{q} P_{q}$, Wigner-sampled initial conditions are selected stochastically from the Wigner distribution obtained from the nuclear density corresponding to Eq. (5). In $R_{q} P_{c l}$ and $R_{c l} P_{q}$, only the "quantum" variable is selected stochastically from a Gaussian distribution in position or in momentum space. Note that, in these two cases, the Gaussian distribution is chosen to be as in the $R_{q} P_{q}$ Wigner sampling. In $R_{c l} P_{c l}$, initial conditions are selected stochastically from a canonical phase-space distribution $\propto \exp -\beta\left[P_{\varphi}^{2} /(2 m)+P_{q}^{2} /\left(2 \omega^{-1}\right)+V_{00}(\varphi, q)\right]$, with

$\beta=\left(k_{B} T\right)^{-1}$ the inverse temperature and $k_{B}$ the Boltzmann constant (we choose here $T=300 \mathrm{~K}$ ). In the thermal Boltzmann sampling, the choice of the temperature is rather arbitrary, because quantum calculations are performed at zero temperature. 

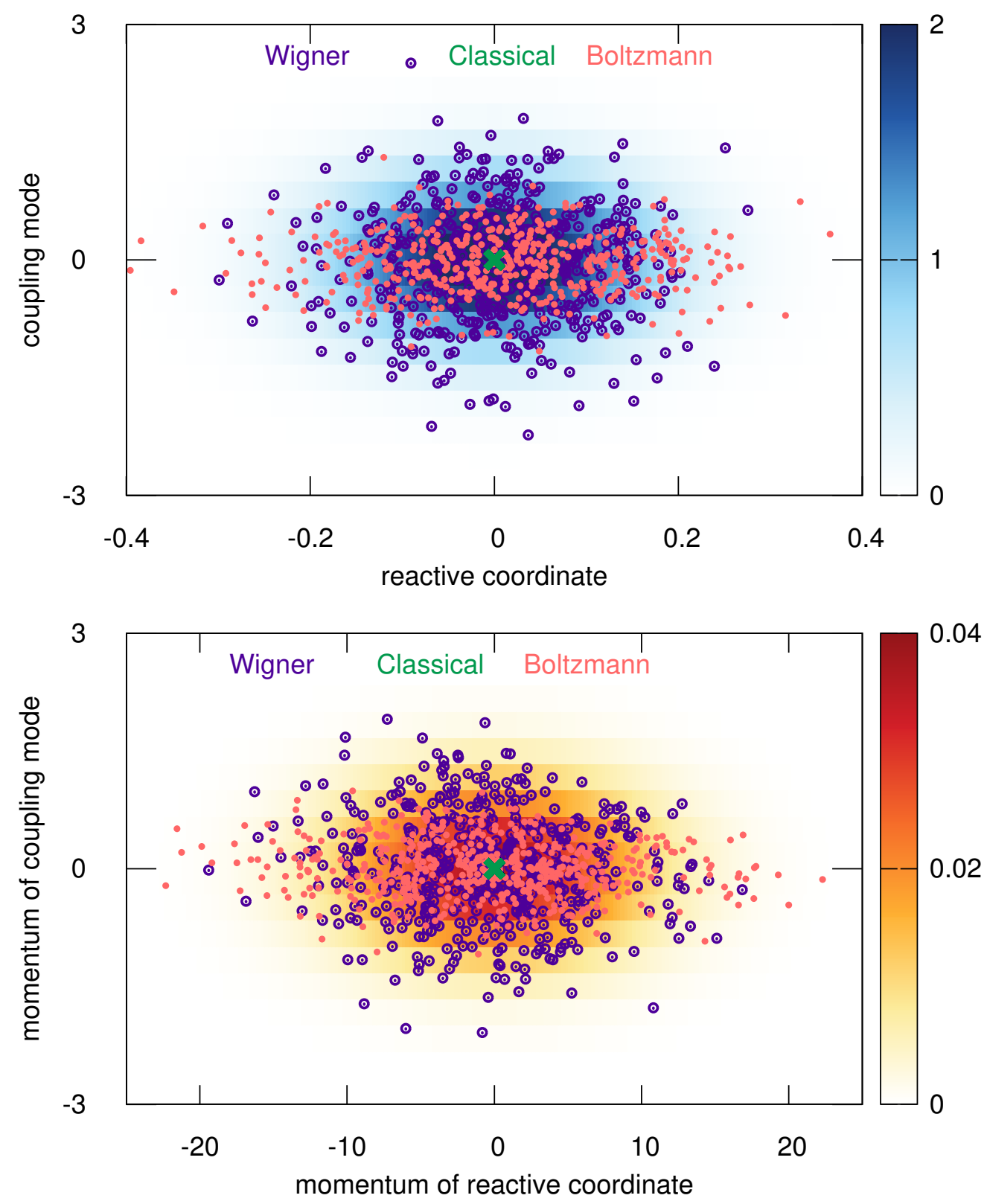

FIG. 1. Initial conditions in position (upper panel) and momentum (lower panel) space. The quantum Wigner distributions are represented by the colored areas (blue for the positions and yellow-red for the momenta). The dots indicate initial conditions used for the trajectories. Purple dots are sampled according to the Wigner distributions (used in $R_{q} P_{q}$, and in the $q$ part of $R_{q} P_{c l}$ and $R_{c l} P_{q}$ ); the green crosses indicate the average position/momentum (used in the $c l$ part of $R_{q} P_{c l}$ and $R_{c l} P_{q}$ ); red dots are sampled according to the Boltzmann distribution at $300 \mathrm{~K}$ (used in $\left.R_{c l} P_{c l}\right)$. 
The four sampling schemes presented above have been chosen based on different physical, but also phenomenological, considerations. Harmonic Wigner and thermal Boltzmann samplings are the most natural choices for selecting initial conditions, according to the literature. Wigner sampling in the harmonic approximation for the ground-state PES is widely used to initialize positions and momenta of classical trajectories before the photo-excitation takes place ${ }^{3,18,93}$. Nonetheless, classical Boltzmann sampling at room temperature has been used as well, even when the initialization concerns trajectories for studies in isolated conditions ${ }^{4,94-97}$, and especially to account for anharmonic effects that are completely neglected within the harmonic Wigner. We believe that the relative importance of quantum effects (treated in the harmonic approximation) and of anharmonicity (treated in the classical approximation), that are clearly system-dependent properties, is a particularly relevant point to account for when choosing an appropriate sampling scheme for initial conditions. This observation underlines the difficulty in identifying an "optimal" sampling scheme for the initialization of the trajectories. Even in the context of a propagation scheme like the quantum-classical Liouville equation, that evolves the partial Wigner distribution, the question might arise, as to whether it is more appropriate to initialize trajectories with one or the other scheme. Indeed, if the system is exactly harmonic or if the anharmonic Wigner distribution is accessible, then the Wigner sampling is preferred, at least in combination with the quantum-classical Liouville equation. The scheme that initializes classical momenta to the same value $P_{c l}$, while Wigner-sampling initial positions, has been chosen because it would be fully consistent with the propagation based on the exact factorization, if the propagation were performed quantum mechanically with (quantum) trajectories ${ }^{27}$. CT-MQC trajectories have been derived from the characteristics of the Hamilton-Jacobi equation, which follows from the nuclear time-dependent Schrödinger equation derived in the framework of the exact factorization (neglecting quantum effects embedded in the quantum potential). In the Hamilton-Jacobi formalism, positions and momenta are not independent variables, as is the case for phase-space variables of the Hamilton formalism. In fact, for each position, the corresponding value of the momentum can be determined as the gradient of the phase of the nuclear wavefunction: for the particular choice of initial condition done in Eq. (5), the gradient of the phase is zero for all positions (as in $R_{q} P_{c l}$ ). Finally, the sampling scheme that initializes classical positions to the same value $R_{c l}$, while Wigner-sampling initial momenta, has been chosen mainly for comparison with the previous scheme.

In Fig. 1, we compare the initial distributions in position (upper panel) and momentum (lower panel) space. The blue-shaded area in the upper panel is the probability density $\left|\chi_{S_{0}}(\varphi, q, t=0)\right|^{2}$ as it is obtained from Eq. (5), whereas the red-shaded area in the lower panel is the momentum distribution associated 
to $\left|\chi_{S_{0}}(\varphi, q, t=0)\right|^{2}$ via the Wigner transform. Therefore, their (uncorrelated) product yields the Wigner distribution. In both panels, the dots represent positions (upper panel) and momenta (lower panel) of the trajectories at the initial time. The indication "Wigner" in Fig. 1 stands for positions/momenta sampled according to the Wigner distribution (blue-shaded or red-shaded areas); the "classical" initial condition means that all positions/momenta are initialized to the same value, indicated as the cross symbol at the origin; "Boltzmann" initial conditions are sampled from a classical canonical distribution at $300 \mathrm{~K}$, and they slightly differ from the Wigner-sampled trajectories, for their widths along the coupling-mode and along the reactive-coordinate directions. Note that, changing the temperature in the Boltzmann sampling would alter (broaden or shrink) the initial distributions, mainly affecting the time-scale of the relaxation process.

The analysis of the effect of the various sampling schemes is based on the study of electronic properties, i.e., the $S_{0}$ and $S_{1}$ populations decomposed into cis and trans contributions, and their coherence, and of nuclear properties, i.e., the nuclear kinetic energy decomposed into contributions arising from the reactive and coupling modes. Before presenting this analysis, let us briefly describe the relaxation process as it is given by quantum vibronic wavepackets.

In Fig. 2, we show the relaxation process at times $75 \mathrm{fs}, 125 \mathrm{fs}$ and $200 \mathrm{fs}$ after the photo-excitation. In the figure, left panels report the nuclear density in the ground state $S_{0}$ (blue areas), whereas right panels present the nuclear density in the excited state $S_{1}$ (red areas). Initially, the wavepacket promoted to $S_{1}$ is localized around $\varphi=0$, and due to the shape on the PES it oscillates towards slightly negative values of $q$ (75 fs). During these oscillations along $q$, the $S_{1}$ wavepacket spreads in $\varphi$ towards the conical intersections (indicated as black dots in all panels). As soon as the conical intersections are reached, density is transferred from $S_{1}$ to $S_{0}(125 \mathrm{fs})$ in a symmetric way along $\varphi$. By construction, the PESs are symmetric along the reactive coordinate. Note that, the system is periodic along the torsion reactive coordinate, thus interference patterns in the ground states are observed at early times (125 fs). Due to the reduced dimensionality of the model, the ground-state wavepacket remains in the conical-intersection region and back-transfer of density from $S_{0}$ to $S_{1}$ is likely to be observed (200 fs).

The dynamics just described is simulated based on the trajectory-based approaches TSH, TSH-EDC, EH and CT-MQC. Results of electronic population, coherence and nuclear kinetic energy are reported below in Sections III A, III B and III C, providing a qualitative analysis of the agreement of quantum-classical calculations based on different sampling schemes of the initial conditions with exact calculations. Note that the observables that are analyzed in the following sections belong to different "classes": electronic populations are not integrated over the whole nuclear configuration space, but are resolved into cis and trans contribu- 

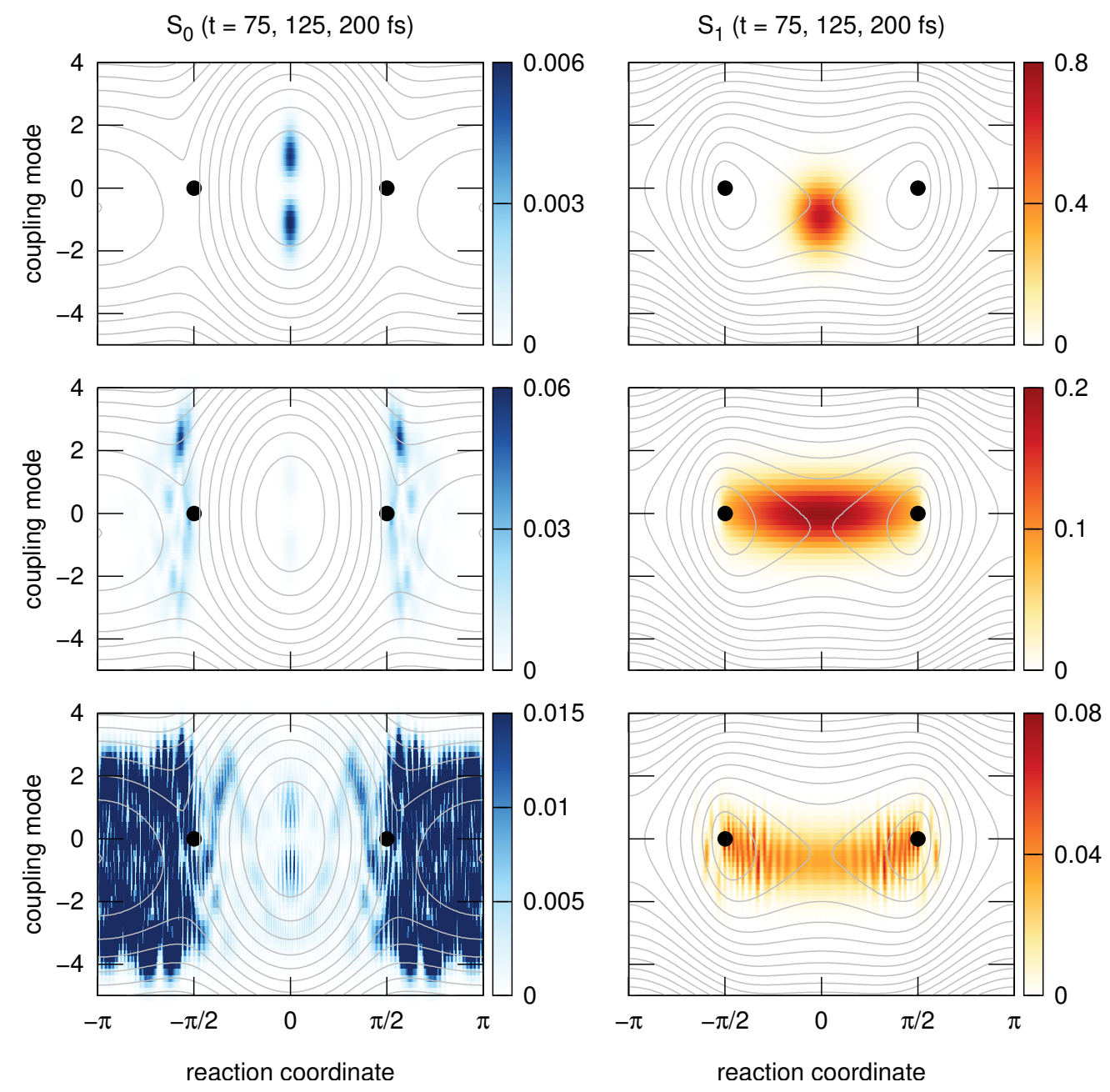

FIG. 2. Snapshots of nuclear dynamics from quantum simulations. Left panels: ground-state $\left(S_{0}\right)$ density as blue-shaded areas. Right panels: excited-state $\left(S_{1}\right)$ density as red-shaded areas. Snapshots are shown at $75 \mathrm{fs}$ (top panels), $125 \mathrm{fs}$ (central panels) and $200 \mathrm{fs}$ (bottom panels) after photo-excitation. The PESs are represented as gray contour plots; the positions of the conical intersections are indicated as black dots.

tions; coherence is quantified based on a indicator that is integrated over the whole configuration space, as well as the nuclear kinetic energy. Afterwards, in Section III D a quantitative analysis is presented, based on the differences of the quantum-classical methods with respect to the reference results.

\section{A. Electronic population analysis}

Electronic populations are decomposed into cis and trans contributions, and are shown for the cis region in Fig. 3 and for the trans region in Fig. 4. Even though cis and trans conformers are strictly defined at the 


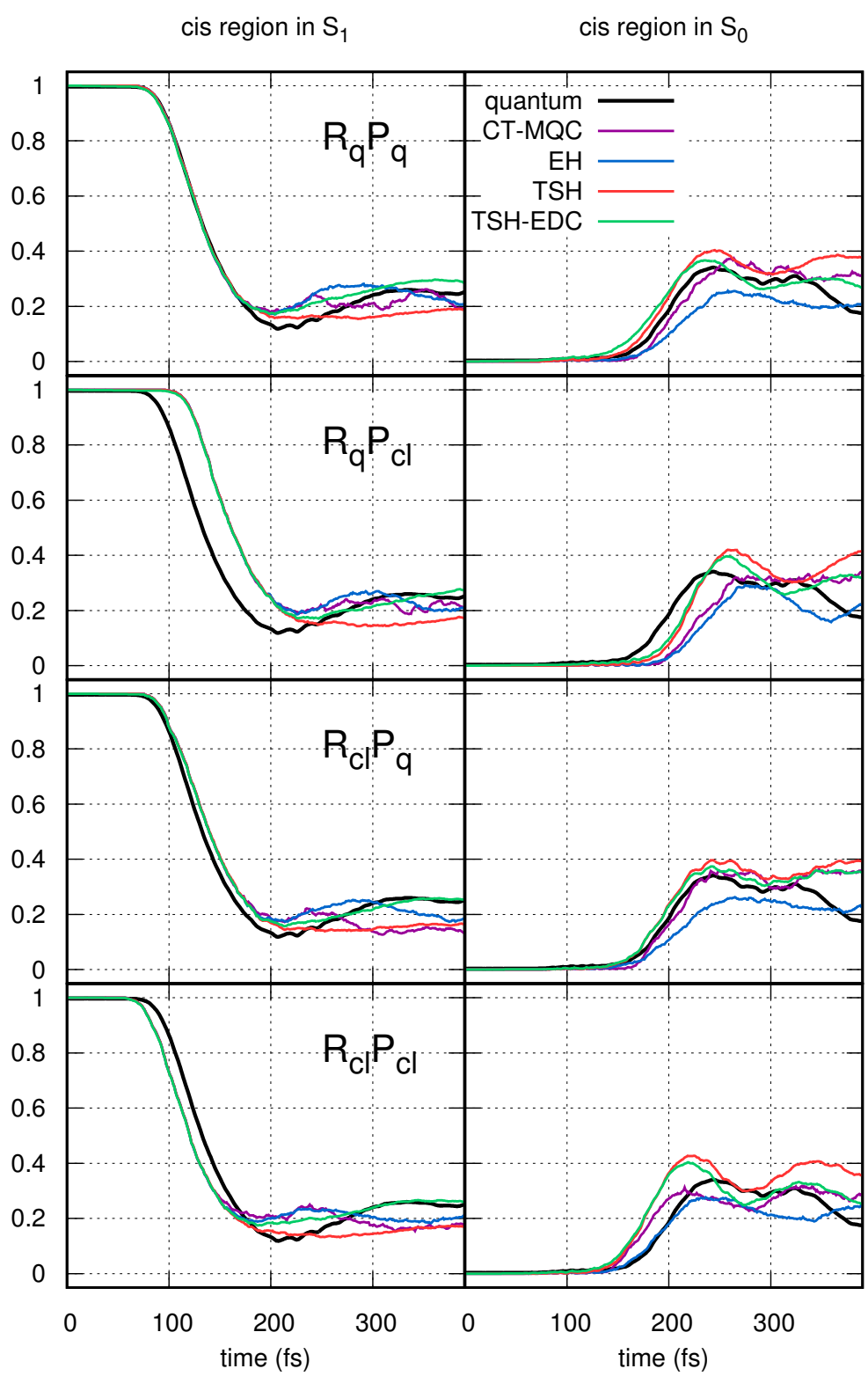

FIG. 3. Probabilities of the cis conformer in the excited state $S_{1}$ (left panels) and in the ground state $S_{0}$ (right panels) as functions of time. Quantum results are indicated in black, TSH results in red, TSH-EDC results in green, EH results in blue and CT-MQC results in purple. Initial conditions for the trajectory-based results are sampled according to the procedures (from top to bottom) $R_{q} P_{q}$, $R_{q} P_{c l}, R_{c l} P_{q}$, and $R_{c l} P_{c l}$, as indicated in each panel.

minima of the PESs, in the discussion below, we refer to the "cis conformer" when the probability density is integrated in the cis region and to the "trans conformer" when the probability density is integrated in the trans region, as indicated in Eqs. (8) and (9). From quantum simulations, we observe an ultrafast decay of the $S_{1}$ population that starts slightly before $100 \mathrm{fs}$. The relaxation process is complete within $200 \mathrm{fs}$, as 


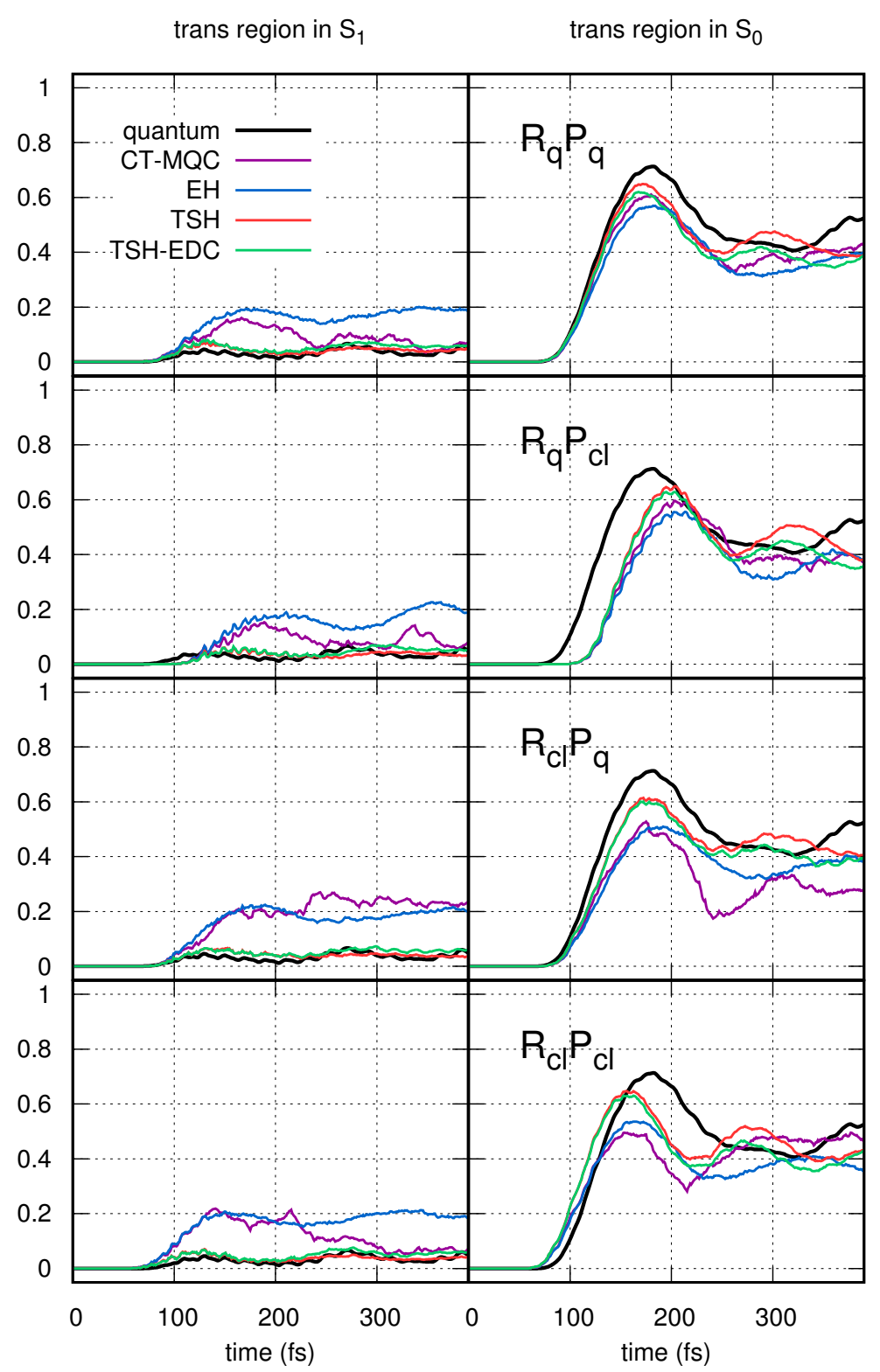

FIG. 4. Probabilities of the trans conformer in the excited state $S_{1}$ (left panels) and in the ground state $S_{0}$ (right panels) as functions of time. The color code and the organization of the plots are the same as in Fig. 3.

the $S_{1}$ population in the cis geometry reaches the value 0.15 . After $200 \mathrm{fs}$, the probability of forming the cis conformer in the excited state increases again. Since the nuclear density is completely delocalized over the available configuration space, and no dissipation channels are considered in the model, at longer times (after $500 \mathrm{fs}$ ) population of the ground and excited state tends to stabilize to an equilibrium value of 0.5 , as observed in Ref. ${ }^{37}$.

Figures 3 and 4 show some general trends: 
- stochastic sampling of the initial momenta appears to be very important. For instance, in the panel $R_{q} P_{c l}$ of Fig. 3-left we observe a delayed offset of the relation process from $S_{1}$ to $S_{0}$ despite the fact that initial positions are randomly distributed. Classical sampling according to $R_{c l} P_{c l}$, as shown by the $S_{1}$ population for the cis conformer (panel $R_{c l} P_{c l}$ of Fig. 3-left), slightly anticipates the relaxation process for all trajectory-based methods, ;

- quantum-classical trajectories sampled according to the $R_{q} P_{c l}$ scheme present high-frequency oscillations between 100 and $200 \mathrm{fs}$ during the increase of the $S_{0}$ population for the trans conformer ( $R_{q} P_{c l}$ panel of Fig. 4-right), signalling an overcoherent transfer of population due to the fact that the momenta are not sampled but they are all initialized to the same value;

- the population of the cis conformer in $S_{1}$ is, qualitatively, well described quantum-classically in the panels $R_{q} P_{q}$ and $R_{c l} P_{q}$ of Fig. 3-left;

- quantum calculations predict that the population of the cis conformer in $S_{1}$ reaches a minimum at $200 \mathrm{fs}$, and increases again towards the value of about 0.25 at $400 \mathrm{fs}$, as shown in the left panels of Fig. 3. However, TSH does not capture this behavior, which is corrected by the decoherence correction in TSH-EDC for all sampling schemes;

- the quantum-classical population of the cis conformer in $S_{0}$ is overall in agreement with quantum results. However, in Fig. 3-right EH tends to underestimate it, and all methods do not capture correctly the final decrease towards the value 0.2 ;

- the population of the trans conformer in $S_{1}$ is well captured, for all initial conditions, only by TSH(EDC), as shown in the left panels of Fig. 4, while it is overestimated by EH in all left panels of Fig. 4 and by CT-MQC for $R_{c l} P_{q}$ at all times;

- the population of the trans conformer in $S_{0}$ is qualitatively well described by all quantum-classical methods, even though the maximum value reached at around $180 \mathrm{fs}$ (see right panels of Fig. 4) is underestimated; the largest deviations are observed for $R_{q} P_{c l}$ and $R_{c l} P_{c l}$ (and in $R_{c l} P_{q}$ by CT-MQC);

- in general, $R_{q} P_{c l}$ and $R_{c l} P_{c l}$ sampling schemes produce larger oscillations than $R_{q} P_{q}$ and $R_{c l} P_{q}$ for all methods, as it is clear in the $S_{0}$ populations for both conformers (right panels of Figs. 3 and 4), suggesting the importance of quantum sampling of the initial momenta to accurately reproduce the relaxation from the excited to the ground state. 
From the analysis of the electronic populations, we can conclude that classical-like sampling of the initial conditions, either without including uncertainty at all (as in $R_{c l} P_{q}$ ) or including it via the Boltzmann distribution (as in $R_{c l} P_{c l}$ ), does not yield unphysical results, as long as the momenta are stochastically distributed. In fact, $R_{c l} P_{q}$ sampling provides for all trajectory-based schemes a good qualitative agreement of the electronic populations with the reference results.

\section{B. Decoherence analysis}

Quantum decoherence is often difficult to capture when trajectory-based approaches to excited-state dynamics are employed. Since a conical-intersection model is considered here, it is interesting to quantify decoherence effects in the present case, and to analyze how well it is reproduced by the quantum-classical methods in comparison to exact dynamics. Therefore, we introduce an indicator of coherence, which has been used in previous studies $4,33,35,98$ and can be computed using the same (analytical) expression based on quantum-dynamics and quantum-classical quantities. The indicator of coherence $\eta(t)$ between the states $S_{0}$ and $S_{1}$ is

$$
\eta(t)=\iint \frac{\left|\chi_{S_{0}}^{*}(\varphi, q, t) \chi_{S_{1}}(\varphi, q, t)\right|^{2}}{|\chi(\varphi, q, t)|^{2}} d \varphi d q \simeq \frac{1}{N_{t r}} \sum_{\alpha=1}^{N_{t r}}\left|\left[C_{S_{0}}^{\alpha}(t)\right]^{*} C_{S_{1}}^{\alpha}(t)\right|^{2}
$$

where the first equality refers to the quantum expression and the second to the quantum-classical expression. The definition of $\eta(t)$ in terms of nuclear wavepackets and in terms of electronic coefficients deserves further clarifications. The vibronic state of the system as function of time can be given in two forms,

$$
|\Psi(\varphi, q, t)\rangle=\chi_{S_{0}}(\varphi, q, t)\left|\phi_{\varphi, q}^{S_{0}}\right\rangle+\chi_{S_{1}}(\varphi, q, t)\left|\phi_{\varphi, q}^{S_{1}}\right\rangle=\chi(\varphi, q, t)\left|\Phi_{\varphi, q}(t)\right\rangle
$$

that are the Born-Huang form ${ }^{19}$ and the exact-factorization form; the latter can be expressed in a BornHuang-like form as done in Eq. (13), without yet using a trajectory notation. The two, fully equivalent, expressions yield the relation between the Born-Huang nuclear wavepacket and the nuclear wavefunction of the exact factorization

$$
\chi_{S_{k}}(\varphi, q, t)=\chi(\varphi, q, t) C_{S_{k}}(\varphi, q, t)
$$

where the electronic coefficients appear. Inserting this result in the quantum-mechanical expression of $\eta(t)$ and making the "classical" substitution $|\chi(\varphi, q, t)|^{2} \rightarrow N_{t r}^{-1} \sum_{\alpha} \delta\left(\varphi-\varphi_{\alpha}(t)\right) \delta\left(q-q_{\alpha}(t)\right)$ yields exactly the quantum-classical expression on the right-hand side of Eq. (eqn: coherence). The indicator of coherence is shown in Fig. 5 for the four propagation methods and for the four initial conditions sampling schemes. 
As soon the $S_{1}$ wavepacket approaches the conical intersection, population is transferred to $S_{0}$, and the non-zero overlap between the two wavepackets is visualized in Fig. 5 as the increase of the value of $\eta(t)$ from zero at around $100 \mathrm{fs}$. At long times, the overlap between the $S_{0}$ and $S_{1}$ wavepackets does not go to zero, because the wavepackets are completely delocalized in configuration space, and $\eta(t)$ does not decay to zero.

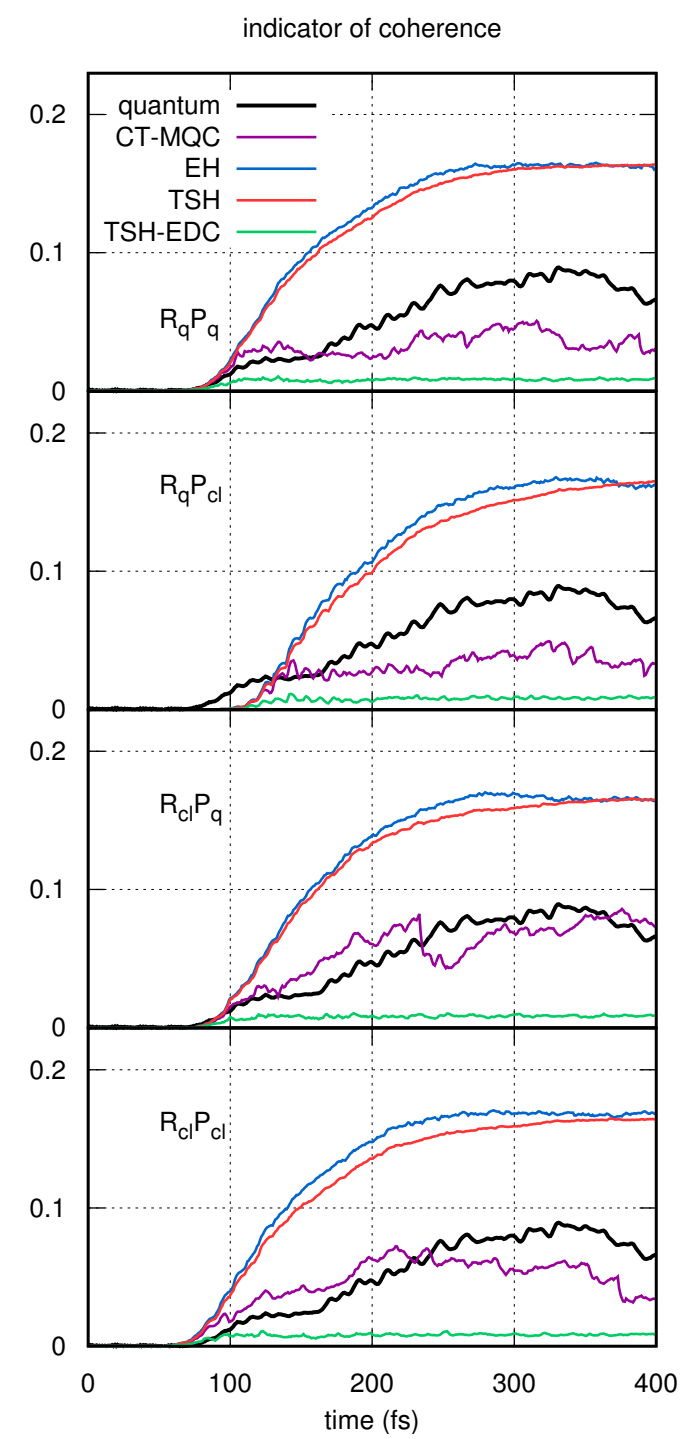

FIG. 5. Indicator of (de)coherence as function of time. The color code and the organization of the plots are the same as in Fig. 3.

In general, as expected, TSH and EH overestimate the coherence, whereas TSH-EDC with the energy parameter used here, i.e., $\mathcal{C}=0.2721 \mathrm{eV}$, overcorrects, and the indicator of coherence is smaller than the quantum-mechanical result. CT-MQC performs fairly well despite the fact that, as discussed in Section II A, 
interference patterns appear in the nuclear wavepacket/density, that are difficult to capture with Gaussians centered at the trajectory positions. Surprisingly, we observe that the decoherence indicator is not strongly affected by the initial sampling, apart from the difference offset (at around $100 \mathrm{fs}$ ) that is related to an anticipated (in $R_{c l} P_{c l}$ ) or delayed (in $R_{q} P_{c l}$ ) decay of the $S_{1}$ population as observed in Fig. 3.

\section{Nuclear kinetic energy analysis}

The nuclear kinetic energy is decomposed into two contributions along the coupling mode $q$ and along the reactive coordinate $\varphi$, and is shown in Fig. 6. In general, we observe coherent oscillations along $q$ as soon as the ground-state wavepacket is promoted to the excited state. The oscillations have a period of about $10 \mathrm{fs}$, and persist for nearly $100 \mathrm{fs}$. Afterwards, the nuclear wavepacket in $S_{1}$ starts losing potential energy as it funnels down the conical intersections, and the kinetic energy increases. The amplitude of the oscillations is damped after $100 \mathrm{fs}$, since the wavepacket loses coherence and spreads in configuration space. The kinetic energy along the reactive coordinate $\varphi$ shows an almost monotonic increase after $50 \mathrm{fs}$, when the nuclear wavepacket in $S_{1}$ spreads towards the conical intersections (and the "molecule" starts rotating towards $\varphi= \pm \frac{\pi}{2}$ ). At later times, when the kinetic energy along the coupling mode seems to reach a stable value of about $0.25 \mathrm{eV}$, the kinetic energy along the reactive coordinate decreases, indication that back-transfer to the excited state (higher potential energy) has started.

As we have shown in previous work ${ }^{37}$, the nuclear kinetic energy gives insightful information on the dynamics, and it appears to be a sensitive tool to estimate the accuracy of approximated methods beyond the standard electronic population analysis that is often reported in the literature. Furthermore, as Fig. 6 shows, the nuclear kinetic energy illustrates very clearly the differences among the sampling schemes tested here. As before, we report some general observations:

- all sampling schemes reproduce the initial oscillations of the nuclear kinetic energy along the coupling mode (Fig. 6-left), but some differences are observed for the phase and the amplitudes of those oscillations;

- only the fully quantum sampling, i.e., $R_{q} P_{q}$, captures exactly the initial oscillations along the coupling mode, as shown in the corresponding panel of Fig. 6-left. The oscillations are out-of-phase in $R_{c l} P_{q}$, they present a larger amplitude in $R_{q} P_{c l}$, and they are centered around a lower energy value in $R_{c l} P_{c l}$, if compared to the reference results; $R_{c l} P_{q}$ and $R_{c l} P_{c l}$ sampling produce oscillations along the coupling mode with smaller amplitude than quantum results; 


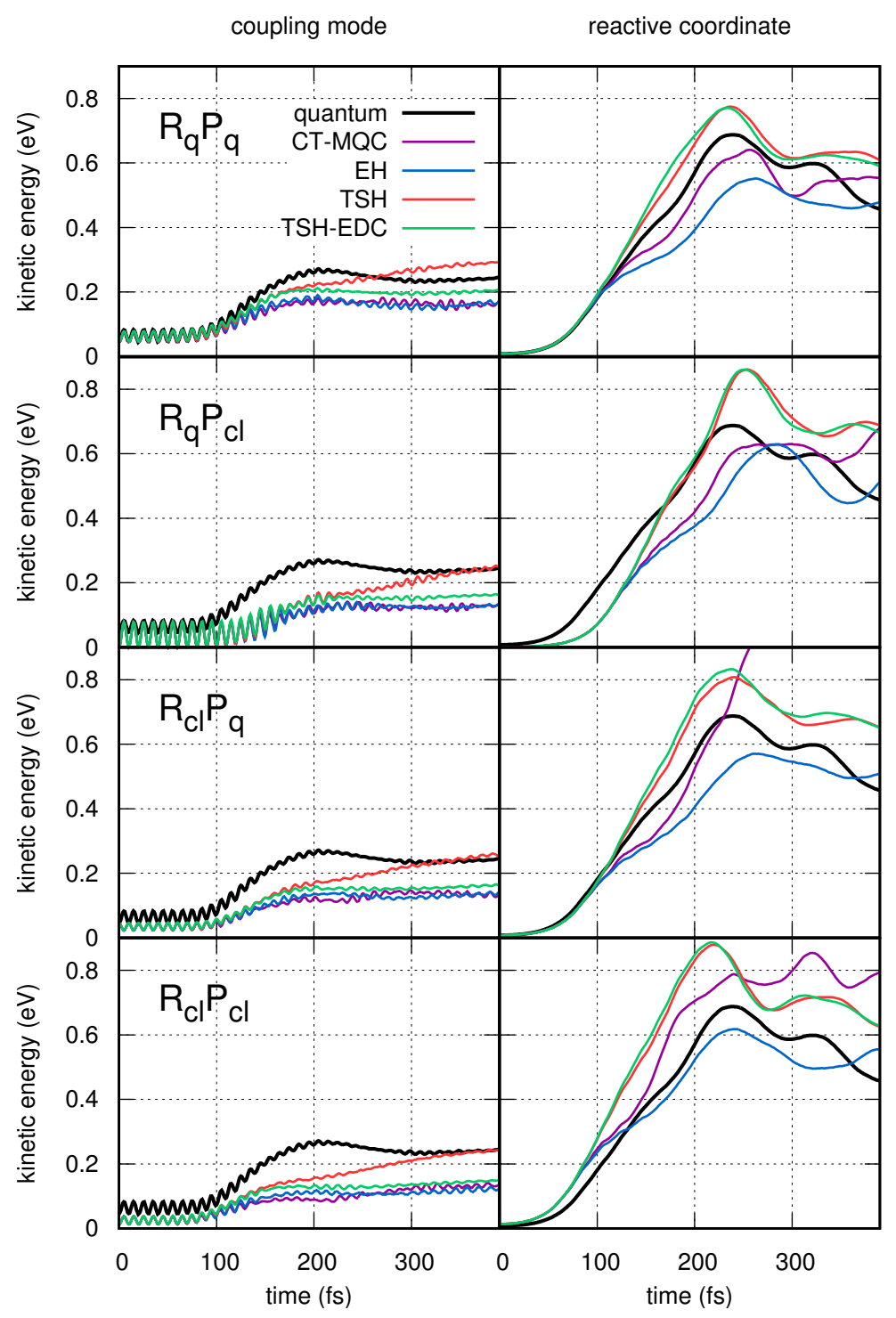

FIG. 6. Nuclear kinetic energies along the coupling mode (left panels) and along the reactive coordinate (right panels) as functions of time. The color code and the organization of the plots are the same as in Fig. 3.

- the nuclear kinetic energy along the coupling mode at long times is underestimated by all methods for all sampling schemes;

- in general, for the kinetic energy along the reactive coordinate (Fig. 6-right), the decrease observed at long times appears to be missing or to be not as clear as in quantum simulations;

- CT-MQC predicts an increasing kinetic energy along the reactive coordinate at long times if initial conditions are sampled according to the $R_{c l} P_{q}$ scheme, whereas, in combination with $R_{q} P_{q}$ and 
$R_{q} P_{c l}$, the energy lies between TSH(-EDC) and EH results;

- in Fig. 6-left, TSH results show a drift a long times for all sampling schemes, which is suppressed by the decoherence correction, as clearly shown for TSH-EDC;

- TSH and TSH-EDC, in combination with all sampling schemes, overestimate the maximum value of the kinetic energy along the reactive coordinate (Fig. 6-right), as also observed in Ref. ${ }^{37}$, while EH systematically underestimate it, and CT-MQC predictions strongly depend on the sampling scheme;

- the kinetic energy along the reactive coordinate is captured qualitative well with $R_{c l} P_{q}$ sampling by TSH(-EDC) and EH.

From the analysis of the nuclear kinetic energy, we can conclude that a classical-like sampling of the initial momenta, as done in $R_{q} P_{c l}$ and $R_{c l} P_{c l}$ does not account for the zero-point energy along the coupling mode, and in fact the plots show that the initial average kinetic energy is lower than the quantum energy. However, those same sampling schemes produce electronic populations in good agreement with quantum results.

In general, all sampling schemes reproduce only qualitatively the behavior of the nuclear kinetic energy in time, but a quantitive agreement is missing across methods. Unexpectedly, $R_{c l} P_{c l}$ does not perform badly, apart from when it is combined with CT-MQC, especially at long times, indicating that a purely classical sampling combined with classical-like evolution equations might be a consistent strategy to performed trajectory-based nonadiabaic simulations.

Capturing energy transfer from the quantum electronic system, that is excited at the initial time with visible light, to the classical nuclear vibrations is a challenging task. One can only guess that, considering a larger number of degrees of freedom, i.e., with the inclusion of an environment in the model ${ }^{9,40,43}$ or by performing atomistic simulations of the whole molecule $2,13,30,99$, might result in a more classical behavior of the whole system, and in a better description of the energy transfer process based on quantum-classical simulations.

\section{Quantitative analysis}

In Sections III A, III B, and III C we presented some results of the quantum and quantum-classical simulations focusing on electronic and nuclear observables that are usually of interest when studying nonadiabatic processes. However, rather than analyzing the physics of the problem at hand, the focus of our 
work is to estimate the effect of various sampling schemes for the trajectories initial conditions in combination with various evolution techniques. Therefore, while Sections III A, III B, and III C provide information about how well the overall process is captured, in a qualitative way, in this section, we aim at a quantitative analysis. Previous sections have shown that, trajectory-based results are in qualitative good agreement with the reference. In fact, in the studied case, the typical electronic energy at play is of the order of $2-3 \mathrm{eV}$, while the nuclear zero-point energy estimated at the cis geometry in the harmonic approximation of the ground-state PES is much smaller, of about $0.19 \mathrm{eV}$ and $0.03 \mathrm{eV}$ for the coupling and reactive coordinates, respectively. Therefore, quantum and classical initial conditions yield comparable results. However, small quantitative differences can be expected, and are indeed observed, as, for instance, the different oscillations of the nuclear kinetic energy along the coupling mode at short times shown in Fig. 6. Those quantitative differences are analyzed in the present section.

Along the trajectories, we compute the difference of the quantum-classical prediction with respect to the expected quantum value, for the seven observables analyzed above; for each simulation technique and for each sampling scheme, we show this difference as function of time in Fig. 7, such that, when this difference is positive, it means that the value is overestimated. The panels in Fig. 7 refer to, from left to right, the $S_{1}$ and $S_{0}$ probabilities for the cis conformer, the $S_{1}$ and $S_{0}$ probabilities for the trans conformer, the indicator of coherence, the kinetic energy along the coupling mode and along the reactive coordinate; the panels, from top to bottom, refer to the different sampling schemes, i.e., $R_{q} P_{q}, R_{q} P_{c l}, R_{c l} P_{q}$ and $R_{c l} P_{c l}$. In each panel, the simulation techniques TSH, TSH-EDC, EH and CT-MQC are compared.

Overall, the differences in the electronic probabilities do not exceed the value \pm 0.2 , in the indicator of coherence the value \pm 0.1 , and the kinetic energy is predicted as well as within $\pm 0.15 \mathrm{eV}$. These observations, combined with the good qualitative agreement between quantum-classical and quantum results, suggests that all methods with all sampling schemes provide meaningful physical information on the process. However, it is evident that the quantum, i.e., Wigner, sampling of initial conditions yields an acceptable quantitive agreement to quantum results across methods and across observables. Even though the sampling scheme $R_{q} P_{c l}$ does not capture correctly the time scale of the initial de-excitation from $S_{1}$ to $S_{0}$ for all evolution techniques, at long times, the differences from quantum results decrease for TSH-EDC and CT-MQC. Note that, the decrease in the difference for the kinetic energy along the coupling mode of TSH at long times might be misleading, since it is the result of the energy drift observed in Fig. 6 (left panels) and not of an actual improvement of the agreement with exact calculations at long times. The sampling scheme $R_{c l} P_{q}$ yields better results than $R_{q} P_{c l}$, especially at short times, even though CT-MQC shows a 

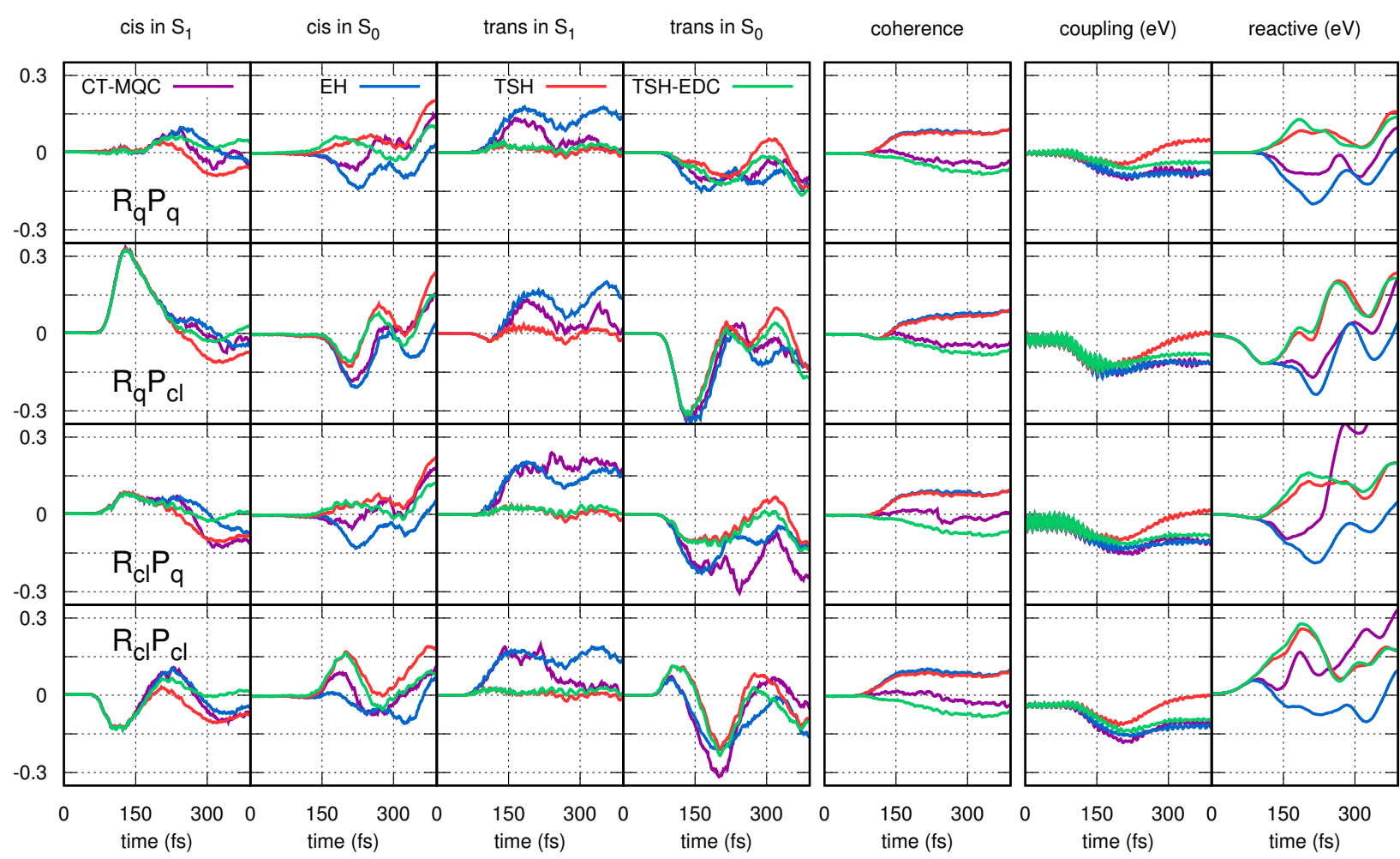

FIG. 7. Differences of trajectory-based and exact results as functions of time. The panels refer to, from left to right, the $S_{1}$ and $S_{0}$ probabilities for the cis conformer, the $S_{1}$ and $S_{0}$ probabilities for the trans conformer, the indicator of coherence, the kinetic energy along the coupling mode and along the reactive coordinate, and, from top to bottom, to the different sampling schemes, i.e., $R_{q} P_{q}, R_{q} P_{c l}, R_{c l} P_{q}$ and $R_{c l} P_{c l}$. In each panel, TSH, TSH-EDC, EH and CT-MQC are compared (the color code is the same as in Fig. 3).

drift in the kinetic energy along the reactive coordinate. Similarly, the thermal sampling $R_{c l} P_{c l}$ performs well in combination with TSH, TSH-EDC and EH, while it appears to be less adequate in combination with CT-MQC.

To further simplify the quantitative analysis of the agreement/deviation of quantum-classical results with the benchmark, quantum calculations, we introduce the following estimate procedure: first, we average the differences with respect to quantum results reported in Fig. 7 over the whole length of the simulations, second, we average the absolute values of the average differences either over the five electronic observables (probabilities and indicator of coherence) or over the two nuclear observables. In Fig. 8, we show how the combinations method-sampling compare to each other, normalizing the results to the largest value, and distinguishing between electronic observables (probabilities and coherences) and nuclear ob- 
servables (energy).

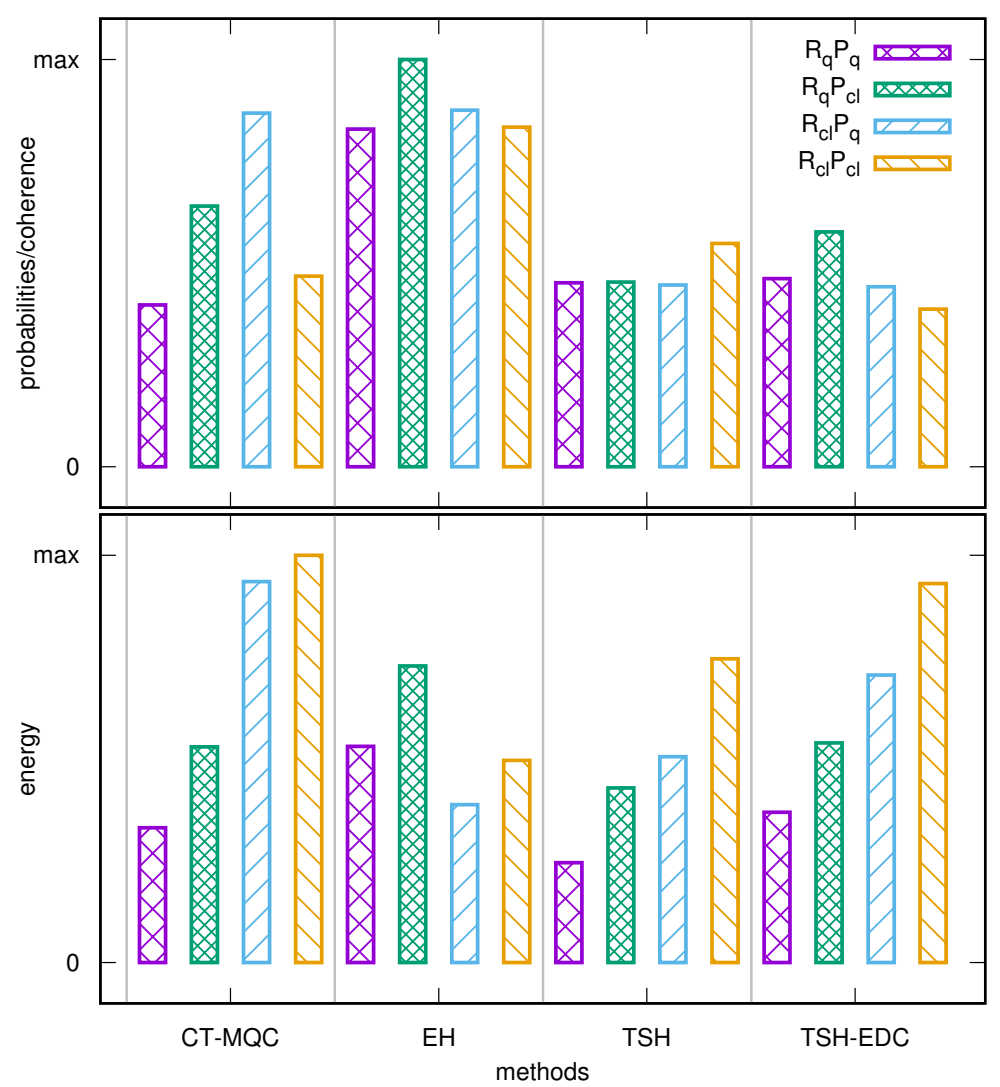

FIG. 8. Comparison among the quantitative agreement of quantum-classical approaches (from left to right, CT-MQC, EH, TSH, TSH-EDC) with quantum results depending on the sampling scheme, averaging, first, the difference of trajectory-based and exact results over the whole length of the trajectories, and, then, averaging the absolute values of the differences either over the five electronic observables (upper panel) or over the two nuclear observables (lower panel). The sampling schemes are indicated as $R_{q} P_{q}$ (purple), $R_{q} P_{c l}$ (green), $R_{c l} P_{q}$ (cyan) and $R_{c l} P_{c l}$ (orange).

Fig. 8 shows that CT-MQC results are quite strongly affected by the choice of the scheme for selecting the initial conditions, and the analysis of the differences suggests that a good quantitative agreement with quantum results is obtained based on the preparation of the initial positions and momenta via a Wigner sampling procedure. Furthermore, the largest error in CT-MQC is obtained for the classical Boltzmann sampling (in the kinetic energy), which might indicate that a quantum sampling strategy is preferable in combination with CT-MQC for consistency with the propagation scheme, since CT-MQC trajectories are coupled and, thus, they preserve some of the quantumness of the nuclear wavepacket. EH results show a different trend if compared to CT-MQC. In fact, in this case, Wigner sampling does not yield the most accu- 
rate estimate of electronic and nuclear observables, and while a purely classical sampling $R_{c l} P_{c l}$ is slightly more accurate for electronic properties, nuclear kinetic energy is better estimated via the $R_{c l} P_{q}$ sampling. Quite surprisingly, the thermal sampling of the initial conditions in combination with EH propagation scheme yields quantitative good results, if compared to a Wigner sampling approach. TSH results are in closer quantitative agreement with quantum results, as the averaged differences are smaller than CT-MQC and $\mathrm{EH}$ (in the best case scenarios). The classical sampling of the initial conditions via $R_{q} P_{c l}$ seems not adequate only for electronic properties, but it performs slightly better than $R_{c l} P_{q}$ and $R_{c l} P_{c l}$ for the nuclear kinetic energy. In general, TSH-EDC improves qualitatively the agreement with exact results, even though this is not highlighted by the quantitative analysis reported in Fig. 8. The sampling scheme $R_{c l} P_{c l}$ seems to better reproduce electronic properties in combination with TSH-EDC than with TSH, however, for the nuclear kinetic energy, the differences with respect to quantum results show the same trend as for TSH.

In general, the good agreement between $R_{q} P_{q}$ sampling and reference results for this model is justified by the shape of the ground-state PES at the cis geometry, where the initial quantum wavepacket is prepared before photo-excitation. At $\varphi=0, q=0$ the $S_{0}$ PES is basically harmonic, thus the vibrational ground state is a Gaussian in $\varphi, q$ (see Eq. (5)) as well as in momentum space. Note that, however, if a system is strongly anharmonic, employing the harmonic approximation to sample the initial Wigner function to account for zero-point energy might be not adequate, and thermal sampling of the Wigner distribution ${ }^{68}$ or of the classical Boltzmann distribution might be preferable ${ }^{69}$.

\section{CONCLUSIONS}

We studied the effect of various sampling schemes for the initial conditions of trajectories in combination with quantum-classical approaches to nonadiabatic dynamics, with the purpose to shed some light on the incorrect energy transfer between electronic and vibrational degrees of freedom observed in Ref. ${ }^{37}$ in a system similar to the one studied here. In Ref. ${ }^{37}$ we ascribed the differences between quantum-classical and exact results mainly to two factors, the delocalized nature of the intersection space of the PESs and the inconsistency between the preparation of the trajectories initial conditions and the subsequent classical dynamics. In the present study, the former issue is somehow removed, because the studied twodimensional model presents a localized conical intersection, which allows us to focus on the quality of the initial sampling. Concerning TSH and TSH-EDC results, we observe similar trend of the nuclear kinetic energy along the reactive coordinate as in Ref. ${ }^{37}$ for all sampling schemes, however, the disagreement with 
respect to quantum results is not as severe as in Ref. ${ }^{37}$. Concerning CT-MQC (with harmonic Wigner and $R_{q} P_{c l}$ samplings) and $\mathrm{EH}$ (with all sampling schemes), the overestimate of the nuclear kinetic energy along the reactive coordinate observed in Ref. ${ }^{37}$ is suppressed. Both observations suggest that in Ref. ${ }^{37}$ the major source of deviation of quantum-classical results from the reference was probably the difficulty of the trajectory-based method to capture dynamics in the presence of the delocalized intersection seam of the three-dimensional model ${ }^{39}$.

We observed that, for the model employed in this work, it might be difficult to reach quantitative agreement between trajectory-based and quantum results, even though the expected behavior in time of the computed observables can be reproduced qualitatively. Some differences have been identified among methods and sampling schemes, and the importance of stochastically sampling the momenta has been highlighted. In all simulation methods studied here, nuclear momenta drive the nonadiabatic population transfer between electronic states. Therefore, averaging over the momenta washes out the details of the population transfer along each trajectory, yielding a better agreement with quantum simulations.

Depending on the computed observable, in the presented examples either electronic populations/coherences or nuclear kinetic energy, a sampling scheme might appear preferable with respect to another, however, a general statement on the adequacy of quantum versus classical sampling approaches in combination with certain quantum-classical techniques cannot be made. Furthermore, our analysis is based on the comparison between exact quantum-dynamical calculations, for which we know exactly what the initial state is, and trajectory-based methods. When it comes to the comparison between trajectory-based results and experiments, the problem of how to choose the initial conditions for the trajectories becomes even more subtle.

It is clear from the points discussed above that, on one hand, a limitation of this study is the transferability of the conclusions. The properties of the system of interest play an important role in the choice of sampling scheme for the initial conditions, as well as in the performance of the trajectory-based method for the dynamics. On the other hand, a general conclusion to be drawn is that different sampling schemes for the initial conditions, when possible, should be tested when aiming to achieve quantitative reliable predictions, even when looking at integrated quantities (over the trajectories). Far from being an exhaustive account of all the possible strategies for sampling the initial conditions and from providing a large studyset of systems, this work points out that the sampling of initial conditions in a nonadiabatic simulations might be as important as the choice of the simulation approach. 


\section{DATA AVAILABILITY STATEMENT}

The data that support the findings of this study are available from the corresponding author upon reasonable request.

\section{DEDICATION}

F.A. would like to dedicate this work to her colleague and friend Serena Bradde in memory of the hard, yet enlightening, time spent together during the undergraduate studies.

\section{ACKNOWLEDGEMENTS}

This work is supported by a public grant overseen by the French National Research Agency (ANR) as part of the "Investissements d'Avenir" program, through the "ADI 2019" project funded by the IDEX Paris-Saclay, ANR-11-IDEX-0003-02.

\section{REFERENCES}

${ }^{1}$ L. M. Ibele and B. F. E. Curchod, "A molecular perspective on tully models for nonadiabatic dynamics," Phys. Chem. Chem. Phys. 22, 15062-15062 (2020).

${ }^{2}$ A. M. Virshup, C. Punwong, T. V. Pogorelov, B. A. Lindquist, C. Ko, and T. J. Martínez, “Photodynamics in complex environments: Ab initio multiple spawning quantum mechanical/molecular mechanical dynamics," J. Phys. Chem. B 113, 3280-3291 (2009).

${ }^{3}$ B. F. E. Curchod and T. J. Martínez, "Ab initio nonadiabatic quantum molecular dynamics," Chem. Rev. 118, 3305-3336 (2018).

${ }^{4}$ S. K. Min, F. Agostini, I. Tavernelli, and E. K. U. Gross, “Ab initio nonadiabatic dynamics with coupled trajectories: A rigorous approach to quantum (de)coherence," J. Phys. Chem. Lett. 8, 3048-3055 (2017).

${ }^{5}$ F. Agostini, B. F. E. Curchod, R. Vuilleumier, I. Tavernelli, and E. K. U. Gross, “TDDFT and quantumclassical dynamics: A universal tool describing the dynamics of matter," in Handbook of Materials Modeling, edited by W. Andreoni and S. Yip (Springer Netherlands, 2018) pp. 1-47.

${ }^{6}$ B. F. E. Curchod, F. Agostini, and I. Tavernelli, "CT-MQC - A coupled-trajectory mixed quantum/classical method including nonadiabatic quantum coherence effects," Euro. Phys. J. B 91, 168 (2018). 
${ }^{7}$ D. Polli, P. Altoè, O. Weingart, K. M. Spillane, C. Manzoni, D. Brida, G. Tomasello, G. Orlandi, P. Kukura, R. A. Mathies, M. Garavelli, and G. Cerullo, "Conical intersection dynamics of the primary photoisomerization event in vision," Nature 467, 440-445 (2010).

${ }^{8}$ S. Gozem, H. L. Luk, I. Schapiro, and M. Olivucci, "Theory and simulation of the ultrafast double-bond isomerization of biological chromophores," Chem. Rev. 117, 13502-13565 (2017).

${ }^{9}$ S. C. Flores and V. S. Batista, "Model study of coherent-control of the femtosecond primary event of vision," J. Phys. Chem. B 108, 6745-6749 (2004).

${ }^{10} \mathrm{~T}$. V. Tscherbul and P. Brumer, “Excitation of biomolecules with incoherent light: Quantum yield for the photoisomerization of model retinal," J. Phys. Chem. A 118, 3100-3111 (2014).

${ }^{11} \mathrm{~T}$. V. Tscherbul and P. Brumer, "Quantum coherence effects in natural light-induced processes: cis-trans photoisomerization of model retinal under incoherent excitation," Phys. Chem. Chem. Phys. 17, 3090430913 (2015).

${ }^{12}$ T. J. Penfold, M. Pápai, K. B. Møller, and G. A. Worth, “Excited state dynamics initiated by an electromagnetic field within the Variational Multi-Configurational Gaussian (vMCG) method," Comput. Theo. Chem. 1160, 24-30 (2019).

${ }^{13}$ M. Filatov, S. K. Min, and K. S. Kim, “Direct nonadiabatic dynamics by mixed quantum-classical formalism connected with ensemble density functional theory method: Application to trans-penta-2,4dieniminium cation," J. Chem. Theory Comput. 14, 4499-4512 (2018).

${ }^{14}$ B. Han and Y. Zheng, "Nonadiabatic quantum dynamics in $\mathrm{O}\left({ }^{3} \mathrm{P}\right)+\mathrm{H}_{2} \rightarrow \mathrm{OH}+\mathrm{H}$ : A revisited study," J. Comput. Chem. 32, 3520-3525 (2011).

${ }^{15} \mathrm{~K}$. Rajak and B. Maiti, “Communications: Direct dynamics study of the $\mathrm{O}\left({ }^{3} \mathrm{P}\right)+\mathrm{C}_{2} \mathrm{H}_{2}$ reaction: Contribution from spin nonconserving route," J. Chem. Phys. 133, 011101 (2010).

${ }^{16}$ W. Hu, G. Lendvay, B. Maiti, and G. C. Schatz, “Trajectory surface hopping study of the $\mathrm{O}\left({ }^{3} \mathrm{P}\right)$ + Ethylene reaction dynamics," J. Phys. Chem. A 112, 2093-2103 (2008).

${ }^{17}$ B. Fu, Y.-C. Han, J. M. Bowman, L. Angelucci, N. Balucani, F. Leonori, and P. Casavecchia, “Intersystem crossing and dynamics in $\mathrm{O}\left({ }^{3} \mathrm{P}\right)+\mathrm{C}_{2} \mathrm{H}_{4}$ multichannel reaction: Experiment validates theory," Proc. Natl. Acad. Sci. 109, 9733-9738 (2012).

${ }^{18}$ R. Crespo-Otero and M. Barbatti, “Recent advances and perspectives on nonadiabatic mixed quantumclassical dynamics," Chem. Rev. 118, 7026-7068 (2018).

${ }^{19}$ F. Agostini and B. F. E. Curchod, “Different flavors of nonadiabatic molecular dynamics,” WIREs Comput. Mol. Sci. 9, e1417 (2019). 
${ }^{20}$ A. J. Jenkins, K. E. Spinlove, M. Vacher, G. A. Worth, and M. A. Robb, “The Ehrenfest method with fully quantum nuclear motion (Qu-Eh): Application to charge migration in radical cations," J. Chem. Phys. 149, 094108 (2018).

${ }^{21}$ F. Agostini, S. K. Min, and E. K. U. Gross, "Semiclassical analysis of the electron-nuclear coupling in electronic non-adiabatic processes," Ann. Phys. 527, 546-555 (2015).

${ }^{22}$ F. G. Eich and F. Agostini, "The adiabatic limit of the exact factorization of the electron-nuclear wave function," J. Chem. Phys. 145, 054110 (2016).

${ }^{23}$ A. Scherrer, F. Agostini, D. Sebastiani, E. K. U. Gross, and R. Vuilleumier, "On the mass of atoms in molecules: Beyond the born-oppenheimer approximation," Phys. Rev. X 7, 031035 (2017).

${ }^{24}$ A. Scherrer, F. Agostini, D. Sebastiani, E. K. U. Gross, and R. Vuilleumier, “Nuclear velocity perturbation theory for vibrational circular dichroism: An approach based on the exact factorization of the electronnuclear wave function," J. Chem. Phys. 143, 074106 (2015).

${ }^{25}$ A. Schild, F. Agostini, and E. K. U. Gross, “Electronic flux density beyond the born-oppenheimer approximation," J. Phys. Chem. A 120, 3316 (2016).

${ }^{26}$ Y. Suzuki and K. Watanabe, "Bohmian mechanics in the exact factorization of electron-nuclear wave functions," Phys. Rev. A 94, 032517 (2016).

${ }^{27}$ F. Talotta, F. Agostini, and G. Ciccotti, "Quantum trajectories for the dynamics in the exact factorization framework: a proof-of-principle test," J. Phys. Chem. A 124, 6764-6777 (2020).

${ }^{28}$ F. Agostini, I. Tavernelli, and G. Ciccotti, “Nuclear quantum effects in electronic (non)adiabatic dynamics," Euro. Phys. J. B 91, 139 (2018).

${ }^{29}$ B. Gu and I. Franco, "Partial hydrodynamic representation of quantum molecular dynamics," J. Chem. Phys. 146, 194104 (2017).

${ }^{30}$ O. Weingart, A. Migani, M. Olivucci, M. A. Robb, V. Buss, and P. Hunt, "Probing the photochemical funnel of a retinal chromophore model via zero-point energy sampling semiclassical dynamics," J. Phys. Chem. A 108, 4685-4693 (2004).

${ }^{31}$ B. F. E. Curchod, F. Agostini, and E. K. U. Gross, “An exact factorization perspective on quantum interferences in nonadiabatic dynamics," J. Chem. Phys. 145, 034103 (2016).

${ }^{32}$ G. Granucci and M. Persico, "Coherent excitation of wavepackets in two electronic states. Interference effects at an avoided crossing," Chem. Phys. Lett. 246, 228-234 (1995).

${ }^{33}$ F. Agostini, "An exact-factorization perspective on quantum-classical approaches to excited-state dynamics," Euro. Phys. J. B 91, 143 (2018). 
${ }^{34}$ J.-K. Ha, I. S. Lee, and S. K. Min, “Surface hopping dynamics beyond nonadiabatic couplings for quantum coherence," J. Phys. Chem. Lett. 9, 1097-1104 (2018).

${ }^{35}$ S. K. Min, F. Agostini, and E. K. U. Gross, “Coupled-trajectory quantum-classical approach to electronic decoherence in nonadiabatic processes," Phys. Rev. Lett. 115, 073001 (2015).

${ }^{36}$ J. E. Subotnik, A. Jain, B. Landry, A. Petit, W. Ouyang, and N. Bellonzi, “Understanding the surface hopping view of electronic transitions and decoherence," Annu. Rev. Phys. Chem. 67, 387-417 (2016).

${ }^{37}$ E. Marsili, M. Olivucci, D. Lauvergnat, and F. Agostini, “Quantum and quantum-classical studies of the photoisomerization of a retinal chromophore model," J. Chem Theory Comput. 16, 6032-6048 (2020).

${ }^{38}$ S. Hahn and G. Stock, "Quantum-mechanical modeling of the femtosecond isomerization in rhodopsin," J. Phys. Chem. B 104, 1146-1149 (2000).

${ }^{39}$ E. Marsili, M. H. Farag, X. Yang, L. D. Vico, and M. Olivucci, “Two-State, Three-Mode Parametrization of the Force Field of a Retinal Chromophore Model," J. Phys. Chem. A 123, 1710-1719 (2019).

${ }^{40}$ S. Hahn and G. Stock, "Femtosecond secondary emission arising from the nonadiabatic photoisomerization in rhodopsin," Chem. Phys. 259, 297-312 (2000).

${ }^{41}$ L. Seidner and W. Domcke, "Microscopic modelling of the photoisomerization and internal-conversion dynamics," Chem. Phys. 186, 27-40 (1994).

${ }^{42}$ M. H. Farag, T. L. C. Jansen, and J. Knoester, "Probing the interstate coupling near a conical intersection by optical spectroscopy," J. Phys. Chem. Lett. 7, 3328-3334 (2016).

${ }^{43}$ D. Dey and N. E. Henriksen, “On weak-field (one-photon) coherent control of photoisomerization," J. Phys. Chem. Lett. 11, 8470-8476 (2020).

${ }^{44}$ B. Balzer, S. Dilthey, S. Hahn, M. Thoss, and G. Stock, "Quasiperiodic orbit analysis of nonadiabatic cis-trans photoisomerization dynamics," J. Chem. Phys. 119, 4204 (2003).

${ }^{45}$ B. F. E. Curchod and F. Agostini, "On the dynamics through a conical intersection," J. Phys. Chem. Lett. 8, 831-837 (2017).

${ }^{46}$ P. E. Videla, A. Markmann, and V. S. Batista, "Floquet study of quantum control of the cis-trans photoisomerization of rhodopsin," J. Chem. Theory Comput. 14, 1198-1205 (2018).

${ }^{47}$ B. Balzer and G. Stock, "Transient spectral features of a cis-trans photoreaction in the condensed phase:? A model study," J. Phys. Chem. A 108, 6464-6473 (2004).

${ }^{48}$ M. Ndong, L. Bomble, D. Sugny, Y. Justum, and M. Desouter-Lecomte, “NOT gate in a cis-trans photoisomerization model," Phys. Rev. A 76, 043424 (2007). 
${ }^{49}$ I. Uspenskiy, B. Strodel, and G. Stock, "Classical description of the dynamics and time-resolved spectroscopy of nonadiabatic cis-trans photoisomerization," Chem. Phys. 329, 109-117 (2006).

${ }^{50}$ B. Balzer, S. Hahn, and G. Stock, “Mechanism of a photochemical funnel: a dissipative wave-packet dynamics study," Chem. Phys. Lett. 379, 351-358 (2003).

${ }^{51}$ S. Hahn and G. Stock, “Ultrafast cis-trans photoswitching: A model study,” J. Chem. Phys. 116, 1085 (2002).

${ }^{52}$ G. Stock, "Classical description of nonadiabatic photoisomerization processes and their real-time detection via femtosecond spectroscopy," J. Chem. Phys. 103, 10015 (1995).

${ }^{53}$ J. C. Tully, “Molecular dynamics with electronic transitions," J. Chem. Phys. 93, 1061 (1990).

${ }^{54}$ G. Granucci and M. Persico, "Critical appraisal of the fewest switches algorithm for surface hopping," J. Chem. Phys. 126, 134114 (2007).

${ }^{55}$ J. C. Tully, “Mixed quantum-classical dynamics," Faraday Discuss. 110, 407 (1998).

${ }^{56}$ F. Agostini, S. K. Min, A. Abedi, and E. K. U. Gross, "Quantum-classical non-adiabatic dynamics: Coupled- vs. independent-trajectory methods," J. Chem. Theory Comput. 12, 2127-2143 (2016).

${ }^{57}$ G. Gossel, F. Agostini, and N. T. Maitra, "Coupled-trajectory mixed quantum-classical algorithm: A deconstruction," J. Chem. Theory Comput. 14, 4513-4529 (2018).

${ }^{58}$ F. Talotta, S. Morisset, N. Rougeau, D. Lauvergnat, and F. Agostini, "Spin-orbit interactions in ultrafast molecular processes," Phys. Rev. Lett. 124, 033001 (2020).

${ }^{59}$ F. Talotta, S. Morisset, N. Rougeau, D. Lauvergnat, and F. Agostini, “Internal conversion and intersystem crossing with the exact factorization," J. Chem Theory Comput. 16, 4833-4848 (2020).

${ }^{60}$ A. Abedi, N. T. Maitra, and E. K. U. Gross, "Exact factorization of the time-dependent electron-nuclear wave function," Phys. Rev. Lett. 105, 123002 (2010).

${ }^{61}$ A. Abedi, N. T. Maitra, and E. K. U. Gross, “Correlated electron-nuclear dynamics: Exact factorization of the molecular wave-function," J. Chem. Phys. 137, 22A530 (2012).

${ }^{62}$ A. Abedi, F. Agostini, Y. Suzuki, and E. K. U. Gross, “Dynamical steps that bridge piecewise adiabatic shapes in the exact time-dependent potential energy surface," Phys. Rev. Lett 110, 263001 (2013).

${ }^{63}$ F. Agostini, E. K. U. Gross, and B. F. E. Curchod, “Electron-nuclear entanglement in the time-dependent molecular wavefunction," Comput. Theo. Chem. 1151, 99-106 (2019).

${ }^{64}$ F. Agostini and E. K. U. Gross, "Exact factorization of the electron-nuclear wavefunction: Theory and applications," in Quantum chemistry and dynamics of excited states: Methods and applications, edited by L. González and R. Lindh (Wiley, 2020) pp. 531-562. 
${ }^{65}$ S. Kube, C. Lasser, and M. Weber, "Monte Carlo sampling of Wigner functions and surface hopping quantum dynamics," J. Comput. Phys. 228, 1947-1962 (2009).

${ }^{66} \mathrm{M}$. Barbatti and K. Sen, "Effects of different initial condition samplings on photodynamics and spectrum of pyrrole," Int. J. Quantum Chem. 116, 762-771 (2015).

${ }^{67}$ R. W. Davies and K. T. R. Davies, "On the Wigner disctribution function for a oscillator," Ann. Phys. 89, 261-273 (1975).

${ }^{68}$ T. Plé, S. Huppert, F. Finocchi, P. Depondt, and S. Bonella, "Sampling the thermal Wigner density via a generalized Langevin dynamics," J. Chem. Phys. 151, 114114 (2019).

${ }^{69}$ N. Klaffki, O. Weingart, M. Garavelli, and E. Spohr, “Sampling excited state dynamics: influence of HOOP mode excitations in a retinal model," Phys. Chem. Chem. Phys. 14, 14299-14305 (2012).

${ }^{70}$ N. A. abd C. Venkataraman and W. H. Miller, "Semiclassical description of electronically nonadiabatic dynamics via the initial value representation," J. Chem. Phys. 127, 084114 (2007).

${ }^{71}$ S. Bonella and D. F. Coker, "LAND-map, a linearized approach to nonadiabatic dynamics using the mapping formalism," J. Chem. Phys. 122, 194102 (2005).

${ }^{72}$ P. Huo and D. F. Coker, "Iterative linearized density matrix propagation for modeling coherent excitation energy transfer in photosynthetic light harvesting," J. Chem. Phys. 133, 184108 (2010).

${ }^{73}$ S. Bonella and D. F. Coker, "Semiclassical implementation of the mapping Hamiltonian approach for nonadiabatic dynamics using focused initial distribution sampling," J. Chem. Phys. 118, 4370 (2003).

${ }^{74}$ T. J. H. Hele and N. Ananth, "Deriving the exact nonadiabatic quantum propagator in the mapping variable representation," Faraday Discuss. 195, 269-289 (2016).

${ }^{75}$ J. Suchan, D. Hollas, B. F. E. Curchod, and P. Slavíček, "On the importance of initial conditions for excited-state dynamics," Faraday Discuss. 212, 307-330 (2018).

${ }^{76} \mathrm{Q}$. Shi and E. Geva, "Semiclassical theory of vibrational energy relaxation in the condensed phase," J. Phys. Chem. A 107, 9059-9069 (2003).

${ }^{77}$ J. Liu and W. H. Miller, “Using the thermal Gaussian approximation for the Boltzmann operator in semiclassical initial value time correlation functions," J. Chem. Phys. 125, 224104 (2006).

${ }^{78}$ J. A. Poulsen, G. Nyman, and P. J. Rossky, "Static and dynamic quantum effects in molecular liquids: A linearized path integral description of water," Proc. Nat. Ac. Sci. 102, 6709-6714 (2005).

${ }^{79} \mathrm{M}$. Basire, D. Borgis, and R. Vuilleumier, “Computing Wigner distributions and time correlation functions using the quantum thermal bath method: application to proton transfer spectroscopy," Phys. Chem. Chem. Phys. 15, 12591 (2013). 
${ }^{80}$ S. C. Althorpe, A. M. Alvertis, W. Barford, R. L. Benson, I. Burghardt, S. Giannini, S. Habershon, S. Hammes-Schiffer, S. Hay, S. Iyengar, A. Kelly, K. Komarova, J. Lawrence, Y. Litman, C. Martens, M. Rossi, K. Sakaushi, D. P. Tew, G. Trenins, R. J. Maurer, D. Plant, A. Schile, S. Sturniolo, and G. Worth, “Zero-point energy and tunnelling: general discussion," Faraday Discuss. 221, 478 (2020).

${ }^{81}$ E. Mangaud, S. Huppert, T. Plé, P. Depondt, S. Bonella, and F. Finocchi, "The fluctuation?dissipation theorem as a diagnosis and cure for zero-point energy leakage in quantum thermal bath simulations," J. Chem. Theory Comput. 15, 2863-2880 (2019).

${ }^{82}$ D. Lauvergnat, “ElVibRot-TnumTana Quantum Dynamics Code," https : / g ithub . com/lauvergn/ ElVibRot-TnumTan.

${ }^{83} \mathrm{H}$. Tal-Ezer and R. R. Kosloff, “An accurate and efficient scheme for propagating the time dependent Schrödinger equation," J. Chem. Phys. 81, 3967-3971 (1984).

${ }^{84}$ R. P. Feynman, “Forces in molecules,” Phys. Rev. 56, 340 (1939).

${ }^{85}$ D. Lauvergnat, "https://github.com/lauvergn/QuantumModelLib/tree/OoP_branch," (2018), QuantumModelLib.

${ }^{86}$ F. Agostini, S. K. Min, I. Tavernelli, and G. H. Gossel, (2018), CTMQC.

${ }^{87}$ S. Garashchuk and V. A. Rassolov, “Energy conserving approximations to the quantum potential: Dynamics with linearized quantum force," J. Chem .Phys. 120, 1181 (2004).

${ }^{88}$ S. Garashchuk and V. A. Rassolov, "Quantum dynamics with Bohmian trajectories: energy conserving approximation to the quantum potential," Chem. Phys. Lett. 376, 358-363 (2003).

${ }^{89}$ S. Garashchuk and V. A. Rassolov, "Semiclassical dynamics with quantum trajectories: Formulation and comparison with the semiclassical initial value representation propagator," J. Chem .Phys. 118, 2482 (2003).

${ }^{90}$ F. Agostini, A. Abedi, Y. Suzuki, S. K. Min, N. T. Maitra, and E. K. U. Gross, "The exact forces on classical nuclei in non-adiabatic charge transfer," J. Chem. Phys. 142, 084303 (2015).

${ }^{91}$ C. L. Lopreore and R. E. Wyatt, “Quantum wave packet dynamics with trajectories," Phys. Rev. Lett. 82, 5190 (1999).

${ }^{92}$ B. Gu, R. J. Hinde, V. A. Rassolov, and S. Garashchuk, "Estimation of the ground state energy of an atomic solid by employing quantum trajectory dynamics with friction," J. Chem. Theory Comput. 11, 2891-2899 (2015).

${ }^{93}$ S. Mai, P. Marquetand, and L. González, "Nonadiabatic dynamics: The SHARC approach," WIREs Comput. Mol. Sci. , e1370 (2018). 
${ }^{94}$ E. Tapavicza, I. Tavernelli, U. Rothlisberger, C. Filippi, and M. E. Casida, “Mixed time-dependent densityfunctional theory/classical trajectory surface hopping study of oxirane photochemistry," J. Chem. Phys. 129, $124108(2008)$.

${ }^{95} \mathrm{G}$. Cui and W. Thiel, “Generalized trajectory surface-hopping method for internal conversion and intersystem crossing," J. Chem. Phys. 141, 124101 (2014).

${ }^{96}$ G. Marchand, J. Eng, I. Schapiro, A. Valentini, L. M. Frutos, E. Pieri, M. Olivucci, J. Léonard, and E. Gindensperger, "Directionality of double-bond photoisomerization dynamics induced by a single stereogenic center," J. Phys. Chem. Lett. 6, 599-604 (2015).

${ }^{97}$ E. Tapavicza, I. Tavernelli, and U. Rothlisberger, “Trajectory surface hopping within linear response timedependent density-functional theory," Phys. Rev. Lett. 98, 023001 (2007).

${ }^{98}$ G. Albareda, A. Kelly, and A. Rubio, "Nonadiabatic quantum dynamics without potential energy surfaces," Phys. Rev. Mat. 3, 023803 (2019).

${ }^{99}$ M. Ruckenbauer, M. Barbatti, T. Müller, and H. Lischka, “Nonadiabatic photodynamics of a retinal model in polar and nonpolar environment," J. Phys. Chem. A 117, 2790-2799 (2013). 